\title{
Constraints on the structure of the core of subgiants via mixed modes: the case of HD 49385
}

\author{
S. Deheuvels ${ }^{1,2}$ and E. Michel ${ }^{1}$ \\ 1 LESIA, UMR8109, Observatoire de Paris, Université Pierre et Marie Curie, Université Denis Diderot, CNRS, 5 place Jules Janssen, \\ 92195 Meudon Cedex, France \\ 2 Department of Astronomy, Yale University, PO Box 208101, New Haven, CT 06520-8101, USA \\ e-mail: sebastien.deheuvels@yale.edu
}

Received 10 May 2011 / Accepted 7 September 2011

\begin{abstract}
Context. The solar-like pulsator HD 49385 was observed with the CoRoT ${ }^{\star}$ satellite over a period of 137 days. The analysis of its oscillation spectrum yielded precise estimates of the mode frequencies over nine radial orders and distinguished some unusual characteristics, such as some modes outside the identified ridges in the échelle diagram and that the curvature of the $\ell=1$ ridge differs significantly from that of the $\ell=0$ ridge.

Aims. We search for stellar models that can reproduce the peculiar features of the oscillation spectrum of HD 49385. After showing that they can be accounted for only by a low-frequency $\ell=1$ avoided crossing, we investigate the information provided by the mixed modes about the structure of the core of HD 49385.

Methods. We propose a toy-model to study the case of avoided crossings with a strong coupling between the p-mode and g-mode cavities in order to establish the presence of mixed modes in the spectrum of HD 49385. We then show that traditional optimization techniques are ill-suited to stars with mixed modes in avoided crossing. We propose a new approach to the computation of grids of models that we apply to HD 49385.

Results. The detection of mixed modes leads us to establish the post-main-sequence status of HD 49385. The mixed mode frequencies suggest that there is a strong coupling between the p-mode and g-mode cavities. As a result, we show that the amount of core overshooting in HD 49385 is either very small $\left(0<\alpha_{\mathrm{ov}}<0.05\right)$ or moderate $\left(0.18<\alpha_{\mathrm{ov}}<0.20\right)$. The mixing length parameter is found to be significantly lower than the solar one $\left(\alpha_{\mathrm{CGM}}=0.55 \pm 0.04\right.$ compared to the solar value $\left.\alpha_{\odot}=0.64\right)$. Finally, we show that the revised solar abundances of Asplund ensure closer agreement with the observations than the classical ones of Grevesse \& Noels. At each step, we investigate the origin and meaning of these seismic diagnostics in terms of the physical structure of the star.

Conclusions. The subgiant HD 49385 is the first star for which a thorough modeling has been attempted to reproduce all the properties of an avoided crossing. It has provided the opportunity to show that the study of the coupling between the cavities in these stars can provide valuable insight into open questions such as core overshooting, the efficiency of convection, and the abundances of heavy elements in stars.
\end{abstract}

Key words. stars: oscillations - stars: evolution - stars: interiors - stars: individual: HD 49385

\section{Introduction}

The existence of mixed modes in the spectrum of stars was first suggested by Scuflaire (1974). By studying non-radial oscillations of highly condensed polytropes, he found waves behaving both as gravity waves in the center, and as acoustic waves in the envelope. These modes were later discovered in the spectrum of $10 M_{\odot}$ models by Osaki (1975), who established that they are associated with avoided crossings between g modes and nonradial $\mathrm{p}$ modes. He showed that whenever the frequencies of two modes of identical degree became close to each other, the modes would avoid each other and exchange natures instead of actually crossing (see Fig. 1). During this exchange, they have a mixed character, similar to those found by Scuflaire (1974). This phenomenon is caused by the evanescent zone that separates the pmode cavity from the g-mode cavity and introduces a coupling between them. Aizenman et al. (1977) gave evidence of that by

* Based on data obtained from the CoRoT (Convection, Rotation and planetary Transits) space mission, developed by the French Space agency CNES in collaboration with the Science Programs of ESA, Austria, Belgium, Brazil, Germany and Spain. decoupling the two cavities and showing that the modes do cross in this case.

Several studies have stressed the great expected potential of avoided crossings in terms of asteroseismic diagnostics. They can indeed provide estimates of the frequency of the g mode that they involve. This is crucial since the g-mode frequencies are determined by the profile of the Brunt-Väisälä frequency in the core and for stars massive enough to have a convective core, this quantity depends to a great extent on the structure of the chemically inhomogeneous zone generated by the withdrawal of the core. This led to the natural idea that the frequency of mixed modes could be used as a means of constraining the amount of overshooting at the boundary of convective cores (e.g., Dziembowski \& Pamyatnykh 1991).

Until now, there have been very few detections of stellar oscillation modes in avoided crossings. Mixed modes were observed in the subgiant star $\eta$ Boo (Kjeldsen et al. 1995), which led to a post-main-sequence classification for the star (Di Mauro et al. 2004; Carrier et al. 2005) and to set an upper limit to the overshooting, but the data were too imprecise to further constrain the internal structure of the star. The recent development of the 


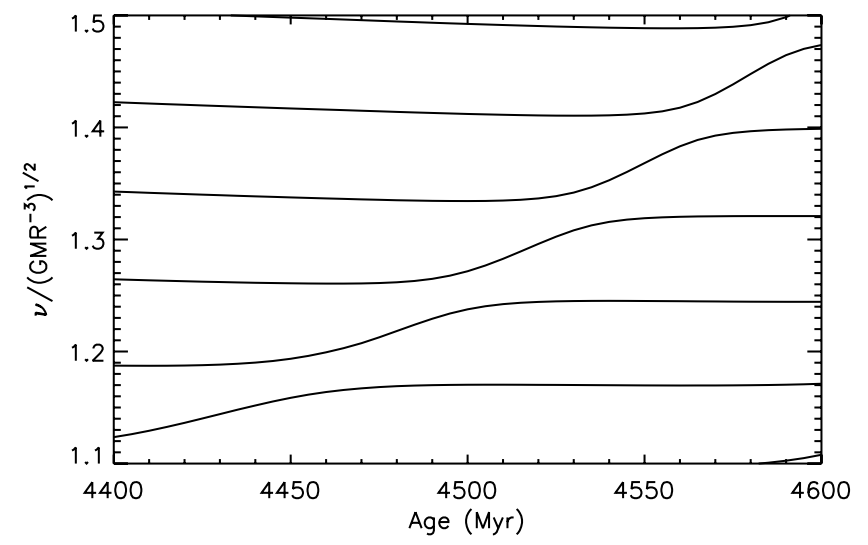

Fig. 1. Evolution of the eigenfrequencies of $\ell=1$ modes as a function of age for a $1.3 M_{\odot}$ model. The frequencies are normalized by the square root of the mean density $\sqrt{G M / R^{3}}$, where $M$ and $R$ are the stellar mass and radius.

space missions CoRoT (Baglin et al. 2006) and Kepler (Koch et al. 2010), by providing long almost uninterrupted time series of high precision photometric data, has opened new opportunities for the detection of mixed modes.

In this paper, we investigate the case of the G0-type star HD 49385, which was observed with the satellite CoRoT over a period of 137 days between October 2007 and March 2008. The analysis of the time series has shown that the star exhibits solar-like oscillations (Deheuvels et al. 2010, further referred to as D10) and the authors could unambiguously identify modes of degree $\ell=0-2$ over nine radial orders. Precise estimates of the mode properties (frequencies, linewidths, amplitudes) were obtained by fitting Lorentzian profiles to the observed spectrum. The authors also pointed out several striking features of the oscillation spectrum of HD 49385. They detected significant peaks that do not follow the expected pattern of high-radial-order p modes. Since HD 49385 is probably an evolved object (judging by its low surface gravity $\log g=4.00 \pm 0.06$, D10) they suggested that some of these peaks might be the signature of mixed modes. The analysis of the oscillation spectrum also showed that the curvature of the $\ell=1$ ridge in the échelle diagram unexpectedly differs from that of the $\ell=0$ ridge at low frequency.

We perform a modeling of HD 49385 based on the spectroscopic and seismic constraints derived for the star by D10. We present in Sect. 2 a preliminary modeling of the star that demonstrates that main-sequence models fail to reproduce the peculiar curvature of the observed $\ell=1$ ridge. We then investigate the possibility that this feature might be caused by mixed modes in the oscillation spectrum of HD 49385. Until now, theoretical studies of avoided crossings have all made the assumption that only two modes are involved and have neglected the contributions of the other modes (e.g., Christensen-Dalsgaard 1981; Gabriel 1980). We study in Sect. 3 the case of avoided crossings when the coupling between the p-mode cavity and the g-mode cavity is too strong to consider this phenomenon as a two-mode only interaction and we show that they can generate a distortion of the ridge that is comparable to the observed one. This leads us to show that the seismic properties of HD 49385 can only be accounted for by the existence of an $\ell=1$ avoided crossing. We then point out in Sect. 4 the limitations of traditional modeling techniques when studying stars with avoided crossings and propose a method to remedy this. This method is applied to search for optimal models of HD 49385 in Sect. 5, with
Table 1. Fundamental parameters of HD 49385 measured from spectroscopic and photometric observations by D10 (top) and estimated using the observed value of $\Delta v$ and $v_{\max }$ (bottom).

\begin{tabular}{lc}
\hline \hline \multicolumn{2}{l}{ Measured parameters } \\
\hline$T_{\text {eff }}(\mathrm{K})$ & $6095 \pm 65$ \\
$(\log g)_{\text {spectro }}$ & $4.00 \pm 0.06$ \\
{$[Z / X](\text { dex })^{\star}$} & $+0.09 \pm 0.05$ \\
$\log \left(L / L_{\odot}\right)$ & $0.67 \pm 0.05$ \\
\hline Parameters estimated from $\Delta v$ and $v_{\max }$ \\
\hline$M / M_{\odot}$ & $1.31 \pm 0.12$ \\
$R / R_{\odot}$ & $1.96 \pm 0.07$ \\
$(\log g)_{\text {seismo }}$ & $3.97 \pm 0.02$ \\
\hline
\end{tabular}

Notes. ${ }^{\star}{ }^{\star}$ The metallicity is defined as $[Z / X] \equiv \log \left[(Z / X) /(Z / X)_{\odot}\right]$.

the aim of determining the information that the frequencies of the mixed modes convey about the structure of the inner regions of HD 49385. In Sect. 6, we discuss these results and investigate their meaning in terms of internal structure and physical processes.

\section{Characteristics of HD 49385 and first step modeling}

\subsection{Observational constraints}

We first give a brief overview of the observational constraints that were derived for this star in previous studies.

\subsubsection{Surface constraints}

The surface observables of HD 49385 were derived by D10 based on a detailed analysis of two high-quality spectra obtained with the NARVAL spectrograph and on the Hipparcos measurement of the star's parallax. We here use their results, which are reproduced in Table 1.

\subsubsection{Seismic constraints}

The star HD 49385 is a solar-like pulsator, which was observed with the CoRoT satellite over a period of 137 days. The ridges of degrees $0 \leqslant \ell \leqslant 2$ were unambiguously identified in the échelle diagram of the power spectrum (D10). The frequencies of these modes were determined over nine radial orders by fitting Lorentzian profiles to the detected modes, using a maximum likelihood estimation.

The star has a mean large separation of $\langle\Delta v\rangle_{\mathrm{obs}}=56.3 \pm$ $0.5 \mu \mathrm{Hz}$ and the frequency at the maximum of the signal is $\nu_{\max }=1010 \pm 10 \mu \mathrm{Hz}$ (the error bars in these measurements were determined based on the method prescribed by Mosser \& Appourchaux 2009). These values can be used to derive a first estimate of the stellar mass and radius using scaling relations. Gai et al. (2011) noted that since the errors in these estimates of the mass and radius are positively correlated, the error we obtain in the quantity $M / R^{2}$ and thus in $\log g$ is small. We report in Table 1 the estimated values of $M, R$, and $\log g$, which are later confronted with those of our best-fit models. We note that the value we obtain for $\log g$ is consistent with the spectroscopic measure of this quantity and has a much smaller error, as expected. 


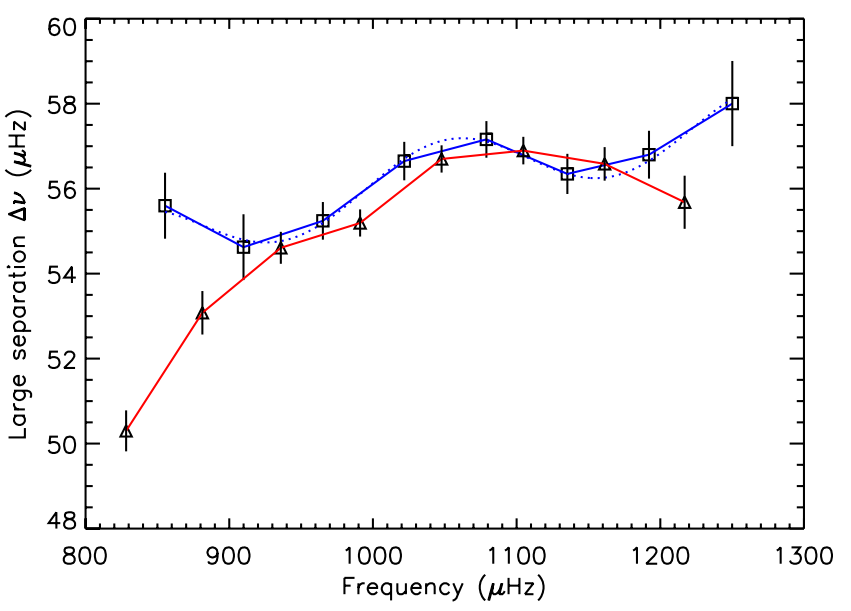

Fig. 2. Profile of the $\ell=0$ (squares and blue line) and $\ell=1$ (triangles and red line) observed large separation represented with 1- $\sigma$ error bars. The blue dotted line shows the result of the least squares fit to a sinusoid of the $\ell=0$ large separation (see text).

A clear oscillation appears in the profile of the $\ell=0$ large separation, as can be seen in Fig. 2. Following the procedure proposed by Roxburgh (2009), we estimate a period of $230 \pm$ $30 \mu \mathrm{Hz}$ for this oscillation. This indicates that the glitch responsible for the oscillation has an acoustic depth of either $\tau_{\text {glitch }} / \tau_{\star}=0.76 \pm 0.04$ or $\tau_{\text {glitch }} / \tau_{\star}=0.24 \pm 0.04$, where $\tau_{\star}$ is the total acoustic radius of the star (we recall that two glitches at acoustic depths of $\tau$ and $\tau_{\star}-\tau$ result in an oscillation of the eigenfrequencies of the same period).

The analysis of the oscillation spectrum of HD 49385 led D10 to identify several unaccounted for features. First, in contrast to expectations for main-sequence solar-like pulsators, the profile of the $\ell=1$ large separation significantly differs from that of the $\ell=0$ large separation, especially in the low-frequency part of the observations (see Fig. 2). In an échelle diagram, this translates into a growing difference between the curvatures of the $\ell=0$ and $\ell=1$ ridges at low frequency. Secondly, several peaks were found to be significant even though they lie outside the identified ridges. One of them, the peak labeled as $\pi_{1}$ by the authors, has a posterior probability that it is caused by noise of as low as $10^{-5}$. It lies close to the $\ell=0$ ridge but not in its direct continuity and it would cause an abrupt step in the $\ell=0$ large separation profile if it were identified as a radial mode. One of the challenges of the modeling of HD 49385 is to understand these peculiarities. We address this question in the following section.

\subsection{Preliminary modeling of HD 49385}

\subsubsection{Properties of the models}

All the models are computed with the stellar evolution code CESAM2K (Morel 1997) and the mode frequencies are derived from them using the Liège Oscillation Code (LOSC, Scuflaire et al. 2008). We used the OPAL equation of state and opacity tables described in Lebreton et al. (2008). The nuclear reaction rates are computed using the NACRE compilation (Angulo et al. 1999). The atmosphere is described by Eddington's grey law and is connected to the envelope at an optical depth of $\tau=10$ to ensure the validity of the diffusion approximation (Morel et al. 1994). We computed models using alternately the mixture of heavy elements of Grevesse \& Noels (1993), which we further refer to as GN93, and the revised mixture of Asplund et al. (2005), referred to as AGS05.

The convective regions are treated using the CanutoGoldman-Mazzitelli (CGM) formalism (Canuto et al. 1996), which involves a free parameter, the mixing length, described as a fraction $\alpha_{\mathrm{CGM}}$ of the pressure scale height. A calibration for the Sun gives $\alpha_{\mathrm{CGM}}=0.64$ (Samadi et al. 2006). In this work, the mixing length is considered as a free parameter.

The radius $R_{\mathrm{cc}}$ of the convective core is determined by the Schwarzschild criterion. Overshooting can be included in the models as an extension of the motion of convective eddies over a distance $d_{\text {ov }}$ outside the core, which is defined as

$d_{\mathrm{ov}} \equiv \alpha_{\mathrm{ov}} \min \left(R_{\mathrm{cc}}, H_{P}\right)$,

where $H_{P}$ is the pressure scale height. In this study, we consider instantaneous mixing in the overshooting region, meaning that we assume that the timescale of the mixing is much smaller than the timescale of the evolution of the star. In this case, the overshooting zone is fully mixed. The temperature gradient is taken to be equal to the adiabatic gradient.

Our models are computed neglecting microscopic diffusion, in order to limit the computational time of our grids of models. However, the effect of microscopic diffusion on our conclusions is studied in Sect. 6.

\subsubsection{Comparison criterion between models and observations}

As is usually done, we compare the stellar models and the observations by computing the merit function $\chi^{2}$ defined as

$\chi^{2} \equiv \sum_{i=1}^{N}\left[\frac{O_{i}^{\mathrm{mod}}-O_{i}^{\mathrm{obs}}}{\sigma_{i}^{\mathrm{obs}}}\right]^{2}$,

where $O_{i}^{\text {obs }}, i=1, N$ represent the $N$ observables selected to constrain the models, $\sigma_{i}^{\text {obs }}$ their error bars, and $O_{i}^{\text {mod }}$ the values of these parameters for the computed models.

To constrain the models, we used the classical observables $T_{\text {eff }}$ and $L / L_{\odot}$. We note that the measured value of $\log g$ could also be used. However, its value is not well enough constrained by spectroscopic measurements to allow it to discriminate among the different models and we therefore no longer mention it in the following. The set of observables is completed by the estimates of the $\mathrm{p}$-mode frequencies of degrees $0 \leqslant \ell \leqslant 2$ obtained by D10. We note that the authors also mentioned the possible detection of two $\ell=3$ modes $\left(\operatorname{modes} \pi_{2}\right.$ and $\left.\pi_{3}\right)$ and proposed estimates of their frequencies. However, given the low signal-to-noise ratio of their profiles, we chose not to include these modes in our set of observables. In addition, one of the $\ell=2$ modes was found to overlap with the closest radial mode $\left(v_{0,14}=855.3 \mu \mathrm{Hz}\right)$. Since none of all the models we computed in this study display this particularity, we assume that this phenomenon is caused by the lower signal-to-noise ratio of the $\ell=2$ modes around the edges of the frequency domain of the oscillations. This $\ell=2$ mode was therefore not included among the observables $O_{i}^{\text {obs }}$.

To compare the eigenfrequencies between models and observations, we know that it is necessary to correct them for the effects of our improper modeling of the structure in the surface layers (e.g., Christensen-Dalsgaard \& Thompson 1997). Kjeldsen et al. (2008) found that in the case of the Sun, the differences 


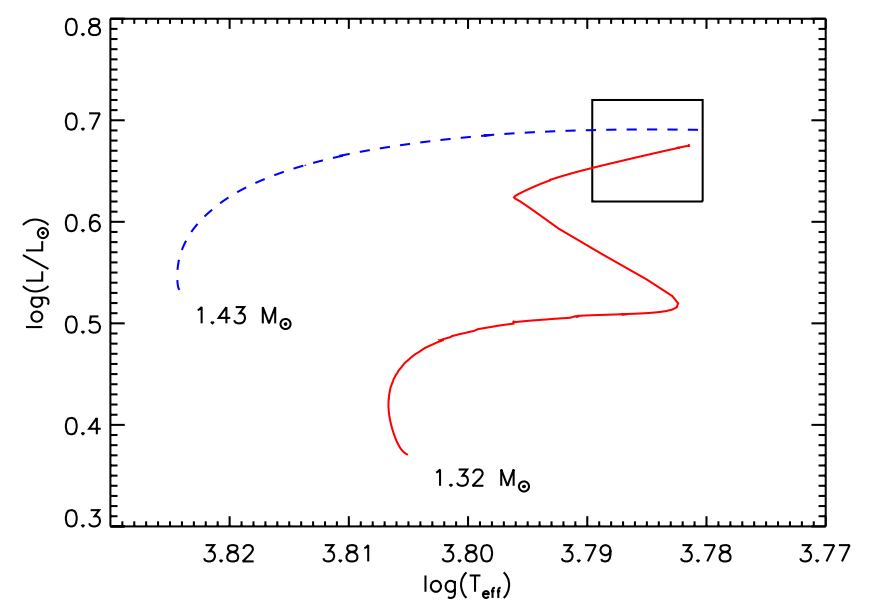

Fig. 3. Evolutionary tracks of two models fitting the position of HD 49385 in the HR diagram. The observed values of the effective temperature and luminosity of HD 49385 are indicated within 1- $\sigma$ error bars by the box. The blue dashed line corresponds to a MS model, and the red solid line to a PoMS model.

between the observed frequencies and those of the best-fit solar model were approximated well by a power law of the form

$v_{n, \ell}^{\mathrm{obs}}-v_{n, \ell}^{\mathrm{best}}=a\left(\frac{v_{n, \ell}^{\mathrm{obs}}}{v_{0}}\right)^{b}$,

where $v_{0}$ is the frequency of the maximum of the signal. The authors tested this law on a few other solar-like pulsators and found reasonable agreement. For this purpose, they assumed that the exponent found in the solar case $(b=4.90)$ could also be used for other targets. We applied a correction of this type to the frequencies of our models. Only our case somewhat differs from that of Kjeldsen et al. (2008): our models are computed using the CESAM $2 \mathrm{~K}$ code and we treat convection with the formalism of Canuto et al. (1996), whereas Kjeldsen et al. (2008) use ASTEC and describe convection using the traditional mixing length theory. It would therefore be irrelevant to use the same exponent as the one found by the authors in the solar case. We computed a solar model matching the set of solar eigenfrequencies found by Gelly et al. (2002). We then fitted the power law given by Eq. (3) to the differences between the observed frequencies and those of our solar model. We obtained a somewhat smaller exponent $(b=4.25)$ which is used to correct the frequencies in the following. We also note that the modes that have a mixed behavior are expected to be less sensitive to the surface effects. To take this into account, for non-radial modes, the surface correction given by Eq. (3) was multiplied by a factor $Q_{n, \ell}^{-1}$, where $Q_{n, \ell}$ corresponds to the ratio of the mode inertia to the inertia of the closest radial mode, as prescribed by Aerts et al. (2010).

\subsubsection{Evolutionary status of HD 49385}

The low value of the spectroscopic $\log g$ obtained for HD 49385 suggests that the star is evolved. We can find models both in the main sequence (hereafter MS) and post main sequence (PoMS) stage, which fit the position of HD 49385 in the HertzsprungRussell (HR) diagram (see Fig. 3). We have a problem that is quite commonplace for evolved objects: a degeneracy between MS models and PoMS models, which results in an uncertainty in the evolutionary stage of the studied object (see e.g., Procyon A in Barban et al. 1999; Provost et al. 2006; $\eta$ Boo in Di Mauro et al. 2003).

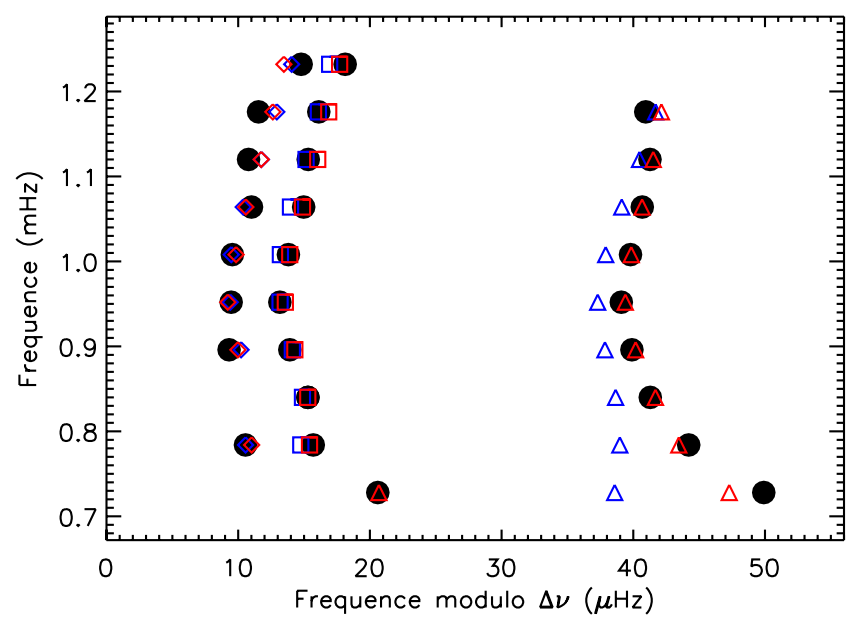

Fig. 4. Échelle diagram of the power spectrum of HD 49385 derived from 137 days of CoRoT data, folded with a mean large separation $\Delta v=56.3 \mu \mathrm{Hz}$. The frequencies of a MS model and a PoMS model are overplotted in blue and red, respectively. The models have been computed to reproduce the observed $\ell=0$ large separation as well as the position of the star in the HR diagram and the PoMS has an $\ell=1$ avoided crossing at low frequency. Squares represent $\ell=0$ modes, triangles $\ell=1$ modes and diamonds $\ell=2$ modes.

The internal structure of these two families of models is very different. The main sequence models are close to the exhaustion of their hydrogen reserves in the center $\left(X_{\mathrm{c}} \sim 0.1\right)$ and they have a small convective core spreading over about $5 \%$ of the stellar radius. The post-main-sequence models are burning hydrogen in a thin layer located above the limit of the convective core that existed during the main sequence stage. There are no longer any convective regions at the center of these models.

\subsubsection{Main-sequence models}

We computed a grid of main sequence models with varying masses, ages, helium abundances, mixing length parameters, and metallicities. We found models that closely fit the observed variations in the $\ell=0$ large separation and the position of the star in the HR diagram. The échelle diagram of one of them is represented in Fig. 4. However, none of the computed MS models are able to reproduce the peculiar curvature of the $\ell=1$ ridge that we mentioned before. The values of the $\chi^{2}$ function that we obtain for MS models are all above 2000. This yields a reduced $\chi^{2}$ above 100 , which indicates a very poor match with the observations. The high value of $\chi^{2}$ for MS models is almost entirely due to the contribution of the $\ell=1$ modes at low frequency (the four $\ell=1$ modes with the lowest frequencies account for more than $90 \%$ of the $\chi^{2}$ value for the best-fit MS models).

In addition, no MS model can explain the presence of the peak $\pi_{1}$. It cannot correspond to the signature of a mixed mode, because MS models that reproduce the surface observables of HD 49385 are not evolved enough to have mixed modes in the frequency domain of the observations. This peak also cannot be identified as an $\ell=0$ mode since the closest radial mode in the models lies about $5 \mu \mathrm{Hz}$ away from it (i.e. at more than $20 \sigma$ ).

\subsubsection{Post-main-sequence models}

In contrast to MS models, some PoMS models that fit the surface parameters of HD 49385 have $\ell=1$ mixed modes in 
the frequency domain of the observations. We considered the possibility that the difference we observe between the curvatures of the $\ell=1$ ridge and the $\ell=0$ ridge might be caused by an $\ell=1$ avoided crossing. To date, all studies of avoided crossings have assumed that only two modes were involved and they neglected the contributions of the other modes (e.g., Christensen-Dalsgaard 1981; Gabriel 1980). In the next section, we investigate the case in which the coupling between the cavities is such that this hypothesis is no longer valid and we try to determine the effect it has on the curvature of the ridge.

\section{Avoided crossings with strong coupling}

\subsection{Analogy with harmonic oscillators}

We now develop an analogy introduced by Deheuvels \& Michel (2010) to gain insight into the characteristics of avoided crossings involving $n$ coupled modes. We recall its main points, for the sake of clarity, and add some details about the choice of the coupling term.

\subsubsection{Avoided crossing with two modes}

Christensen-Dalsgaard (2003), based on the work of von Neuman \& Wigner (1929), proposed a simple analogy that captures the main aspects of avoided crossings between two modes. He considered the two cavities of the star as two coupled harmonic oscillators $y_{1}(t)$ and $y_{2}(t)$, responding to the system of equations

$$
\begin{aligned}
& \frac{\mathrm{d}^{2} y_{1}(t)}{\mathrm{d} t^{2}}=-\omega_{1}(\lambda)^{2} y_{1}+\alpha y_{2}, \\
& \frac{\mathrm{d}^{2} y_{2}(t)}{\mathrm{d} t^{2}}=-\omega_{2}(\lambda)^{2} y_{2}+\alpha y_{1},
\end{aligned}
$$

where $\alpha$ is the coupling term between the two oscillators and $\omega_{1}(\lambda), \omega_{2}(\lambda)$ are the eigenfrequencies of the uncoupled oscillators (in the particular case where $\alpha=0$ ). They were chosen to depend on a parameter $\lambda$, which was used to model the change in the dimensions of the cavities as the star evolves. We assume that for a certain $\lambda=\lambda_{0}$, the frequencies of the uncoupled oscillators cross, i.e. $\omega_{1}\left(\lambda_{0}\right)=\omega_{2}\left(\lambda_{0}\right) \equiv \omega_{0}$.

Solving Eq. (4), we obtain the two solutions

$$
\omega_{ \pm}^{2}=\frac{\omega_{1}^{2}+\omega_{2}^{2}}{2} \pm \frac{1}{2} \sqrt{\left(\omega_{1}^{2}-\omega_{2}^{2}\right)^{2}+4 \alpha^{2}} .
$$

If the coupling term $\alpha$ is very small relative to the difference between the eigenfrequencies $\left(\alpha \ll\left|\omega_{1}^{2}-\omega_{2}^{2}\right|\right)$, then the eigenfrequencies of the system are close to $\omega_{1}$ and $\omega_{2}$. If, on the other hand, $\left|\omega_{1}^{2}-\omega_{2}^{2}\right| \ll \alpha_{1,2}$, then the eigenfrequencies can be approximated by

$$
\omega_{ \pm}^{2}=\omega_{0}^{2} \pm \alpha
$$

To analyze these solutions, we choose the eigenfrequencies of the uncoupled oscillators $\omega_{1}(\lambda)$ and $\omega_{2}(\lambda)$, such that they simulate a p mode and a $g$ mode. With reference to Fig. 1, we define $\omega_{1}(\lambda)=1$ and $\omega_{2}(\lambda)=\lambda$. In this case, an avoided crossing occurs in the system around $\lambda_{0}=1$. The variations in the two eigenfrequencies $\omega_{ \pm}(\lambda)$ are shown in Fig. 5. The two modes clearly exchange natures during the avoided crossing and the intensity of the phenomenon depends on the strength of the coupling between the oscillators.

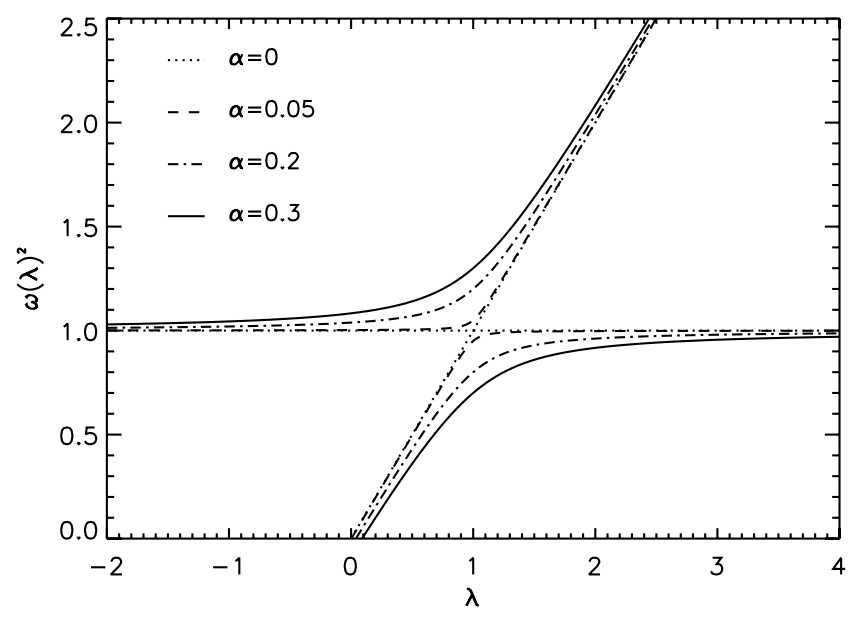

Fig. 5. Variations in the eigenfrequencies $\omega_{ \pm}$of the system with parameter $\lambda$, for different values of the coupling term $\alpha$.

\subsubsection{Avoided crossing with $n$ modes}

The analogy described in Sect. 3.1.1 holds if two modes only are affected during an avoided crossing: this corresponds to neglecting the coupling between the two considered modes and the other modes in the spectrum. We now push the analogy a step further by assuming that these other coupling terms play a significant role. We consider $n$ oscillators, instead of only two. We choose $n-1$ of them to simulate high-radial-order $\mathrm{p}$ modes, by giving them equidistant eigenfrequencies that are constant with $\lambda$. The last oscillator simulates a g mode. We define

$$
\begin{aligned}
& \omega_{i}(\lambda)=\omega_{i-1}(\lambda)+\Delta \omega, \text { for } i=2, \ldots, n-1 \\
& \omega_{n}(\lambda)=\lambda
\end{aligned}
$$

The numerical values of $\omega_{1}(\lambda)$ and $\Delta \omega$ (1 and 0.1 respectively) are chosen to roughly match Fig. 1.

We then introduce a coupling $\alpha_{i}$ between the $\mathrm{g}$ mode and the $i$ th $\mathrm{p}$ mode. Normally, the $\mathrm{p}$ modes should also be coupled to each other. However, since their eigenfrequencies remain equidistant at all times, we can neglect these coupling terms in our analogy. As $\lambda$ increases, the $\mathrm{g}$ mode will experience successive avoided crossings with all the $\mathrm{p}$ modes. According to Eq. (6), the deviation of the eigenfrequency very close to the $i$ th avoided crossing is $\delta \omega \sim \alpha_{i} /\left(2 \omega_{i}\right)$. To ensure that all the avoided crossings have a comparable intensity (as seems to be roughly the case in the models, see Fig. 1), we choose the different coupling terms $\alpha_{i}$ such that

$\alpha_{i}=\gamma \omega_{i}$,

where $\gamma$ describes the strength of the coupling between the pmode cavity and the g-mode cavity. We obtain the system of equations

$$
\begin{aligned}
& \frac{\mathrm{d}^{2} y_{1}(t)}{\mathrm{d} t^{2}}=-\omega_{1}^{2} y_{1}+\gamma \omega_{1} y_{n} \\
& \vdots \\
& \frac{\mathrm{d}^{2} y_{n-1}(t)}{\mathrm{d} t^{2}}=-\omega_{n-1}^{2} y_{n-1}+\gamma \omega_{n-1} y_{n} \\
& \frac{\mathrm{d}^{2} y_{n}(t)}{\mathrm{d} t^{2}}=-\omega_{n}^{2} y_{n}+\gamma \omega_{1} y_{1}+\ldots+\gamma \omega_{n-1} y_{n-1} .
\end{aligned}
$$




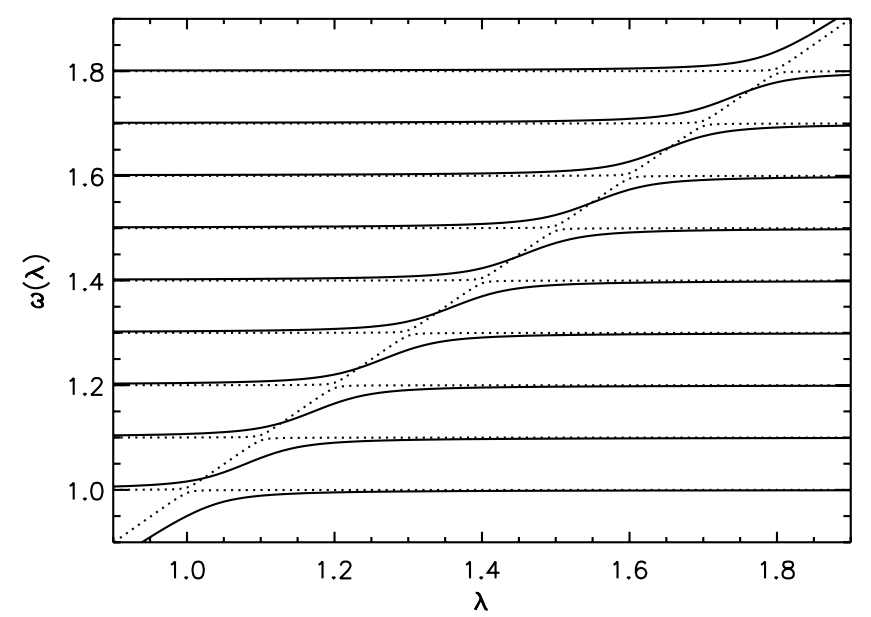

Fig. 6. Variations in the eigenfrequencies of $(n-1) \mathrm{p}$ modes coupled to a g mode, which undergoes avoided crossings with the $\mathrm{p}$ modes (here, $n=10)$. The dashed lines correspond to a "weak coupling" $(\gamma=0.01)$, and the full lines to a "strong coupling" $(\gamma=0.06)$.

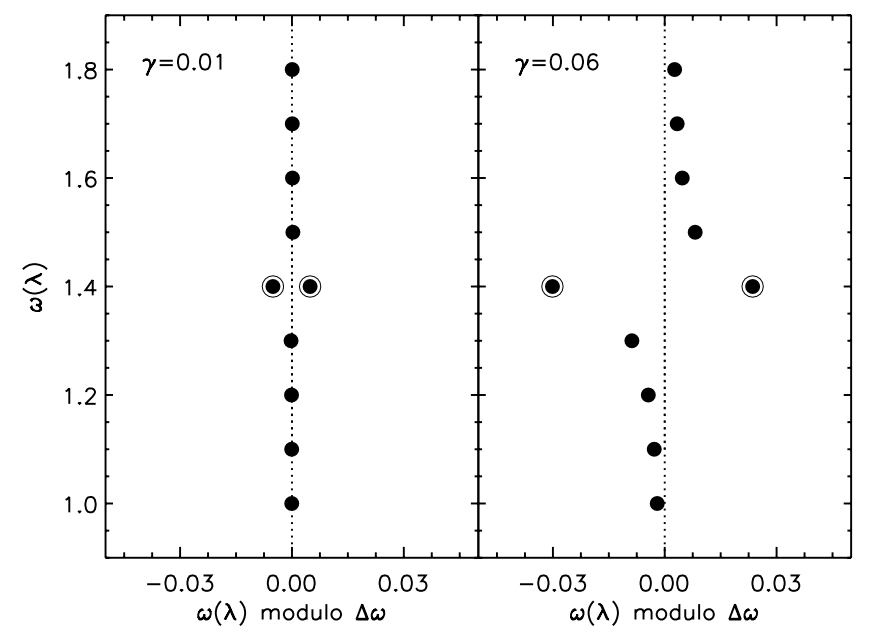

Fig. 7. Échelle diagrams of the eigenfrequencies of ten coupled harmonic oscillators, at a given "time" $\lambda$ in the vicinity of an avoided crossing. The left panel presents the case of a weak coupling $(\gamma=0.01)$ and the right panel the case of a strong coupling $(\gamma=0.06)$. The frequencies that are circled correspond those used to estimate the strength of the coupling in Sect. 6 .

By writing the different oscillators $y_{i}(t)=c_{i} \exp (-\mathrm{i} \omega t)$, the eigenfrequencies of the system are found by solving the eigenvalue problem $A C=\omega^{2} C$ with

$A=\left[\begin{array}{ccccc}\omega_{1}^{2} & 0 & \cdots & 0 & -\gamma \omega_{1} \\ 0 & \omega_{2}^{2} & & \vdots & -\gamma \omega_{2} \\ \vdots & & \ddots & & \vdots \\ 0 & \cdots & & \omega_{n-1}^{2} & -\gamma \omega_{n-1} \\ -\gamma \omega_{1} & \cdots & & -\gamma \omega_{n-1} & \omega_{n}^{2}\end{array}\right]$

and $C=\left[c_{1}, \ldots, c_{n}\right]$.

The solution is plotted in Fig. 6 for two different values of $\gamma$. For each value of the parameter $\lambda$, we plot an échelle diagram with the eigenfrequencies of the coupled system (Fig. 7). For weak coupling, the ridge remains straight and the approximation that only two modes are affected by the avoided crossing is legitimate. However, for strong coupling, more $\mathrm{p}$ modes in the neighborhood of the avoided crossing have a mixed behavior and the ridge is distorted as shown in Fig. 7.

\subsection{Comparison with stellar models}

In a star, the aforementioned coupling is due to the existence of an evanescent zone between the two cavities: the wider this zone, the weaker the coupling. Since the Lamb frequency increases with the dergee $\ell$, the coupling is stronger for modes of small degrees. We present in Fig. 8a the propagation diagram of a $1.3 M_{\odot}$ model, which is sufficiently evolved to have lowdegree avoided crossings (post main sequence). For this model, the ridges of the modes of degrees $1 \leqslant \ell \leqslant 3$ are shown in an échelle diagram in Fig. 8b. Clearly, for modes of degree $\ell \geqslant 2$, the currently adopted hypothesis that only two modes are affected by the avoided crossing is valid, the rest of the ridge being almost unaltered. However, for the $\ell=1$ avoided crossing, the coupling is strong enough to distort the ridge in a way that is very comparable to the one we obtained in our simple analogy (bottom panel of Fig. 7).

\subsection{Application to the case of HD 49385}

By fine-tuning the mass and age of PoMS models, we managed to find a model with an $\ell=1$ avoided crossing in the low-frequency part of the frequency range of the observations (around $750 \mu \mathrm{Hz}$ ) and that also reproduces the observed mean value of the large separation (we present in Sect. 4 a method to find these models in a systematic way). The échelle diagram of this model is represented in Fig. 4. We observe that the curvature of the $\ell=1$ ridge is far more accurately reproduced by this model than by MS models. Interestingly, we note that by adjusting the age of the model, the frequency of the mode that behaves mainly as a $g$ mode in the avoided crossing matches the frequency of the aforementioned peak $\pi_{1}$.

We conclude that the observed oscillation spectrum of HD 49385 can be satisfactorily reproduced only if an $\ell=1$ avoided crossing causes a distortion in the $\ell=1$ ridge of the échelle diagram. We thus obtain a firm detection of mixed modes in the spectrum of the star. We note that the avoided crossing occurs at the far bottom of the frequency range of the observations, which explains why we only detect the upper half of the ridge distortion described in Sect. 3. We are also able to identify the mode that has a mainly g-mode behavior in the avoided crossing (corresponding to the peak $\pi_{1}$ ). This is very valuable when conducting a more thorough modeling of the star (see Sect. 5), since this mode is the most sensitive to the center of the star. Finally, the detection of an $\ell=1$ avoided crossing in the spectrum of HD 49385 enables us to establish that the star is in a PoMS stage.

\section{Stellar modeling using the avoided crossing: potential and strategy}

\subsection{Potential of avoided crossings with strong coupling}

Mixed modes have a high seismic diagnostic value because they are sensitive to the structure of the most central regions of the stars, which remains poorly understood.

The frequencies of mixed modes depend on the profile of the Brunt-Väisälä frequency in the stellar core. To understand the information which we can expect to derive from them, it is 
S. Deheuvels and E. Michel: Constraints on the structure of the core of HD 49385 via mixed modes
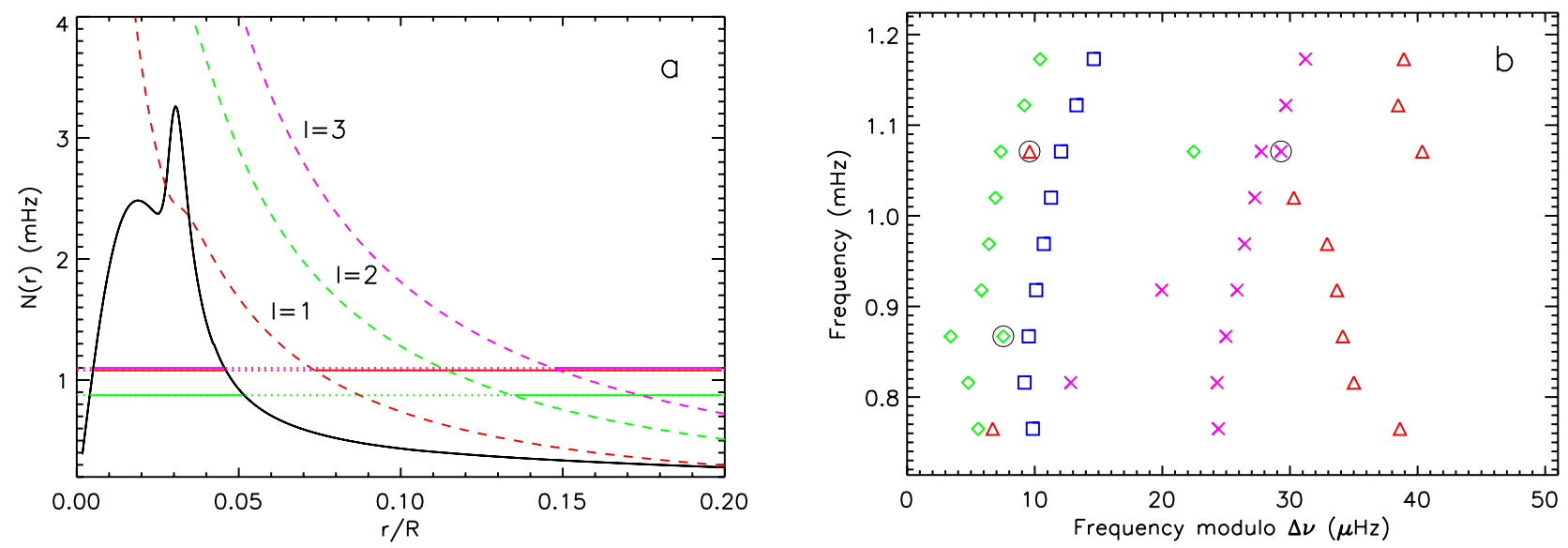

Fig. 8. Left: propagation diagram of a $1.3 M_{\odot}$ post-main-sequence model. The Brunt-Väisälä frequency is represented by the full black line and the Lamb frequencies by the dashed lines $(\ell=1 \mathrm{red}, \ell=2$ green, $\ell=3$ purple). The propagation regions of three mixed modes of degrees $\ell=1,2$, and 3 are represented by the full colored lines and their evanescent regions by the dotted colored lines. Right: échelle diagram of the same model ( $\ell=0$ blue squares, $\ell=1$ red triangles, $\ell=2$ green diamonds, $\ell=3$ purple crosses). The mixed modes represented in the left panel are circled.

convenient to write the Brunt-Väisälä frequency as a function of the gradients

$\nabla_{\mathrm{ad}}=\left.\frac{\partial \ln T}{\partial \ln P}\right|_{S}, \quad \nabla=\frac{\partial \ln T}{\partial \ln P}, \quad \nabla \mu=\frac{\partial \ln \mu}{\partial \ln P}$,

where $P, T$ and $\mu$ correspond to the stellar pressure, temperature and mean molecular weight, respectively. The subscript $S$ indicates that the definition is valid for constant entropy. The Brunt-Väisälä frequency can be divided in two different contributions

$N^{2}=\frac{g}{H_{P}}\left(\nabla_{\mathrm{ad}}-\nabla\right)+\frac{g}{H_{P}} \nabla \mu$,

where $g$ is the gravity inside the star and $H_{P}$ is the local pressure scale-height. The first term in the right hand side of Eq. (13) depends on the temperature stratification. The right term characterizes the dependence on the gradient of mean molecular weight $\nabla \mu$. These two components are shown in Fig. 9 for the model presented in Sect. 3.2.

Two different features of avoided crossings with strong coupling provide information about the structure at the center of the star:

1. The frequency at which the avoided crossing occurs. The knowledge of the frequency of an avoided crossing provides an estimate of the frequency of the g mode that it involves. This opportunity is extremely important, because the observation of pure g modes in main sequence stars is currently impossible. In the framework of the asymptotic theory (Tassoul 1980), the frequency of a g mode of radial order $n$ and degree $\ell$ can be approximated by

$\omega_{n, \ell} \sim \frac{\sqrt{\ell(\ell+1)}}{(n-1 / 2) \pi} \int_{r_{1}}^{r_{2}} \frac{N}{r} \mathrm{~d} r$

where $r_{1}$ and $r_{2}$ are the mode turning points in the g-mode cavity. In the following, we will consider avoided crossings involving low-radial-order g modes, for which the asymptotic approximation is no longer valid. However, their frequencies mainly depend on $\int_{r_{1}}^{r_{2}} N \mathrm{~d} r / r$. A measurement of the frequency of a $\mathrm{g}$ mode provides information about the structure of the g-mode cavity $\left(r_{1} \leqslant r \leqslant r_{2}\right.$ in Fig. 9). In particular, the g-mode frequency is sensitive to the peak

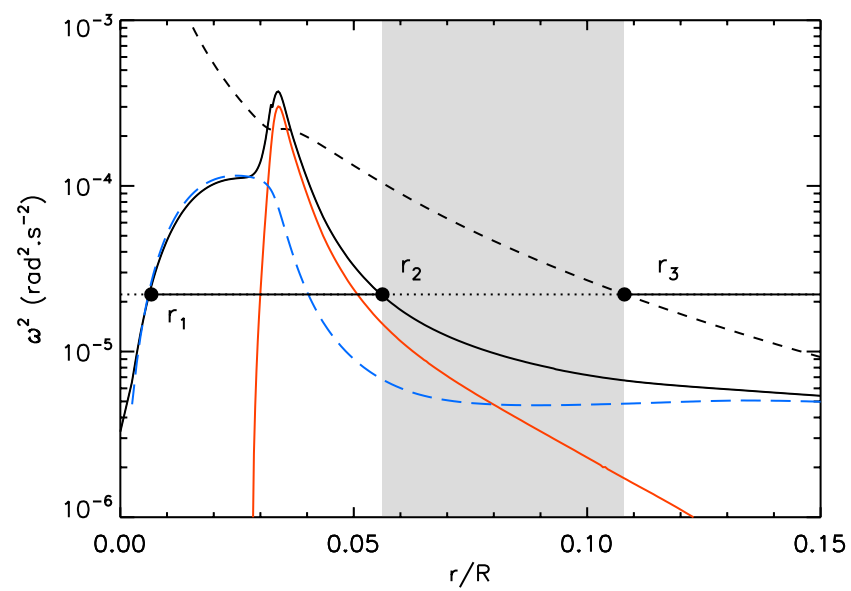

Fig. 9. Propagation diagram for a $1.3 M_{\odot}$ post-main-sequence model with an $\ell=1$ avoided crossing. The Brunt-Väisälä frequency (black solid line) can be separated in two contributions: the part linked to the temperature stratification (long-dashed blue line) and the one linked to the gradient of mean molecular weight (red solid line). The Lamb frequency for $\ell=1$ modes is represented by the black dashed line. The shaded area indicates the evanescent zone. The radii $r_{1}$ and $r_{2}$ correspond to the turning points of the g-mode cavity and $r_{3}$ the turning point of the p-mode cavity.

left in the profile of $\nabla \mu$ by the withdrawal of the convective core, for stars massive enough to have had such a core during the main sequence. This is the reason why the detection of mixed modes is expected as a means of constraining the amount of overshooting at the boundary of convective cores (e.g., Dziembowski \& Pamyatnykh 1991).

2. The intensity of ridge distortion. We showed in Sect. 3 that more than two modes are affected by $\ell=1$ avoided crossings. The modification to their frequencies (characterized by a distortion in the $\ell=1$ ridge in the échelle diagram) depends on the strength of the coupling between the p-mode cavity and the g-mode cavity. This coupling mainly depends on the profile of the Brunt-Väisälä frequency in the evanescent zone: the higher $N(r)$ in this region, the stronger the coupling. The intensity of the ridge distortion should therefore provide information about the structure of the star in the evanescent zone (i.e. in the region $r_{2} \leqslant r \leqslant r_{3}$ in Fig. 9), 
which complements those given by the frequency of the avoided crossing. We illustrate this complementarity with the modeling of the star HD 49385 in Sects. 5 and 6.

\subsection{Limitations of traditional optimization techniques to model stars with avoided crossings}

As mentioned in our introduction, there have been numerous theoretical studies of avoided crossings. However, very few studies have tried to fit stellar models to the properties of an avoided crossing. The first reason is that until very recently, the spectra for which the detection of mixed modes was claimed were of too low quality to perform such an investigation. The second reason is that avoided crossings occur on a very short timescale (typically of the order of $1 \mathrm{Myr}$ or less) compared to the stellar evolution timescale, which causes some inherent difficulties. The usual approach to modeling a star consists of computing a grid of models with a wide range of stellar parameters (mass, age, etc.) to find the optimal model that minimizes the $\chi^{2}$ function defined in Eq. (2). Applying this procedure with a time step of the order of the avoided crossing timescale is infeasible, because it would require the computation of a tremendously large number of models. On the other hand, with a larger time step, we have a very low probability of finding models that correctly reproduce the frequency of the avoided crossing and we therefore miss the best-fit models. We note that it would also be impractical to bluntly apply an automatic minimization using for instance the algorithm of Levenberg-Marquardt, as prescribed by Miglio \& Montalbán (2005). This technique is based on the computation of the Hessian matrix at each iteration and thus requires us to define a step for the time derivatives. The gap between the stellar evolution timescale and that of the avoided crossing makes it impossible to find a satisfactory time step for the procedure.

\subsection{Narrowing down the dimensions of the model space}

We now try to adapt the traditional grid-of-model approach to the special case of targets for which mixed modes are detected. For this purpose, we show that we can overcome the obstacles we have just mentioned by no longer considering the stellar age and mass as free parameters of our fit.

\subsubsection{Relation between $v_{\text {cross }}$ and the stellar age}

The frequency at which an avoided crossing occurs (denoted $v_{\text {cross }}$ ) corresponds to the frequency of the uncoupled $\mathrm{g}$ mode that it involves and is therefore linked to the profile of the Brunt-Väisälä frequency in the core (see Sect. 4.1). Figure 10 shows the variations in the frequencies of the first two uncoupled g modes during the evolution of a $1.3 M_{\odot}$ model (the sharp variations in the mode eigenfrequencies around the age of $4050 \mathrm{Gyr}$ in this plot correspond to the transition between the MS stage and the PoMS stage).

During the main sequence, the first term in the right hand side of Eq. (13) nearly vanishes at the center because the temperature gradient is almost adiabatic in the convective core. The g-mode cavity essentially corresponds to the chemically inhomogeneous zone left by the withdrawal of the core. At this point, the Brunt-Väisälä frequency is still low, so that low-degree $\mathrm{g}$ modes do not reach the frequency range of high-order $\mathrm{p}$ modes and no avoided crossing can be observed.

At the end of the main sequence, when the hydrogen reserves are exhausted in the core, the star is left with an almost

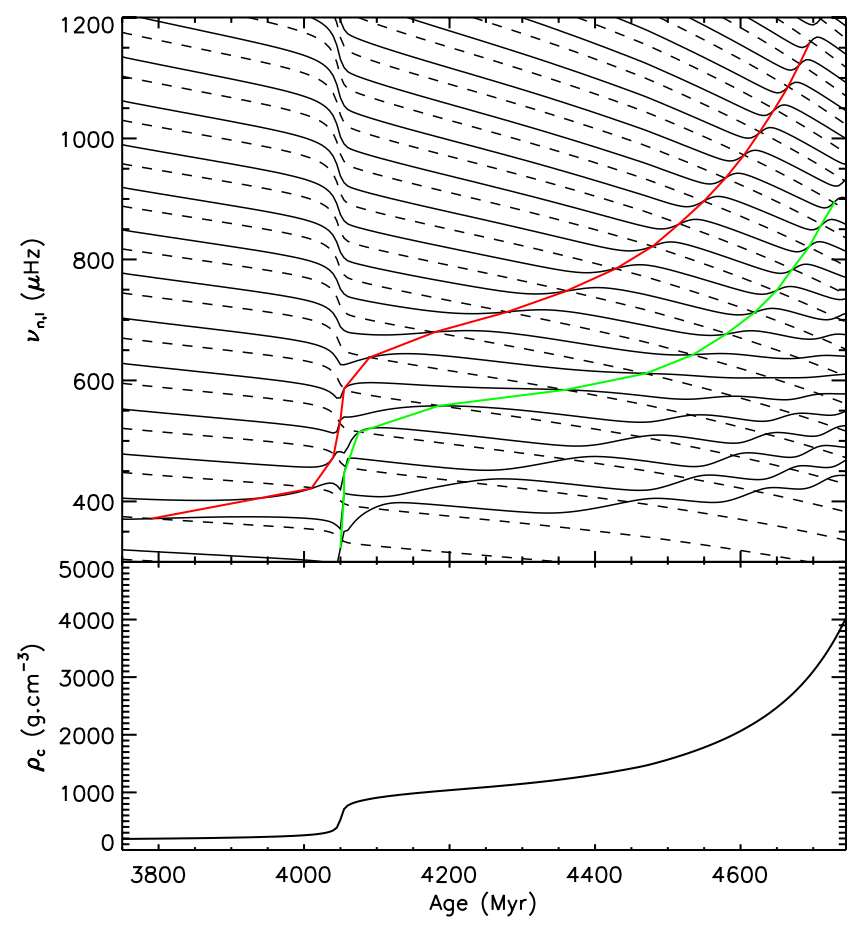

Fig. 10. Top: evolution of the frequencies of $\ell=0$ modes (dashed black lines) and $\ell=1$ modes (full black lines) with age for a $1.3 M_{\odot}$ model. The frequencies of the first two $\ell=1$ uncoupled g modes are also represented ( $g_{1}$ mode in red and $g_{2}$ mode in green). Bottom: evolution of the central density $\rho_{\mathrm{c}}$ with age for the same model.

isothermal helium core. The inner regions contract until nuclear reactions are triggered in the layers where hydrogen remains. The evolution of the Brunt-Väisälä frequency is then mainly determined by the value of the central density $\rho_{\mathrm{c}}$. To explain this, we note from Eq. (13) that $N$ depends on the factor $g / H_{P}$, which can also be written $\rho g^{2} / P$. In the most central regions, the gravity term can be approximated as $g(r) \sim G \rho_{\mathrm{c}} r$. In that case, we have

$\frac{g}{H_{P}} \sim \frac{\rho_{\mathrm{c}}^{3}}{P_{\mathrm{c}}} r^{2} \sim \frac{\rho_{\mathrm{c}}^{2} \mu_{\mathrm{c}}}{T_{\mathrm{c}}} r^{2}$,

where $P_{\mathrm{c}}$ and $T_{\mathrm{c}}$ are the central pressure and temperature. The models show that the central temperature hardly changes in the isothermal core during evolution in the post-main-sequence phase. The mean molecular weight remains constant because the chemical composition in the core does not vary. In contrast, the contraction of the star causes the central density to increase by more than one order of magnitude relative to its value at the end of the MS, as can be seen in the lowest panel of Fig. 10. As a result, the factor $g / H_{P}$ and thus the Brunt-Väisälä frequency mainly depend on the evolution of the central density $\rho_{\mathrm{c}}$. This is confirmed by Fig. 10, which shows the tight relation between the evolution of $v_{\text {cross }}$ and $\rho_{\mathrm{c}}$ for a $1.3 M_{\odot}$ model.

We conclude that the avoided crossing frequencies monotonically increase during the evolution of the star. We can therefore find for every model (with fixed mass and physics) one stellar age such that the avoided crossing occurs at the same frequency as in the observations. For the model represented in Fig. 10, an age of $4374 \pm 0.8 \mathrm{Myr}$ satisfies this condition (the error bar is determined by requiring a fit of $v_{\text {cross }}^{\text {obs }}$ within a $1-\sigma$ error bar). The precision we obtain is such that we can consider the age to be entirely determined by the value of $v_{\text {cross }}$ (for models of a given mass and physics) and we can cease to consider the age as a free 
S. Deheuvels and E. Michel: Constraints on the structure of the core of HD 49385 via mixed modes
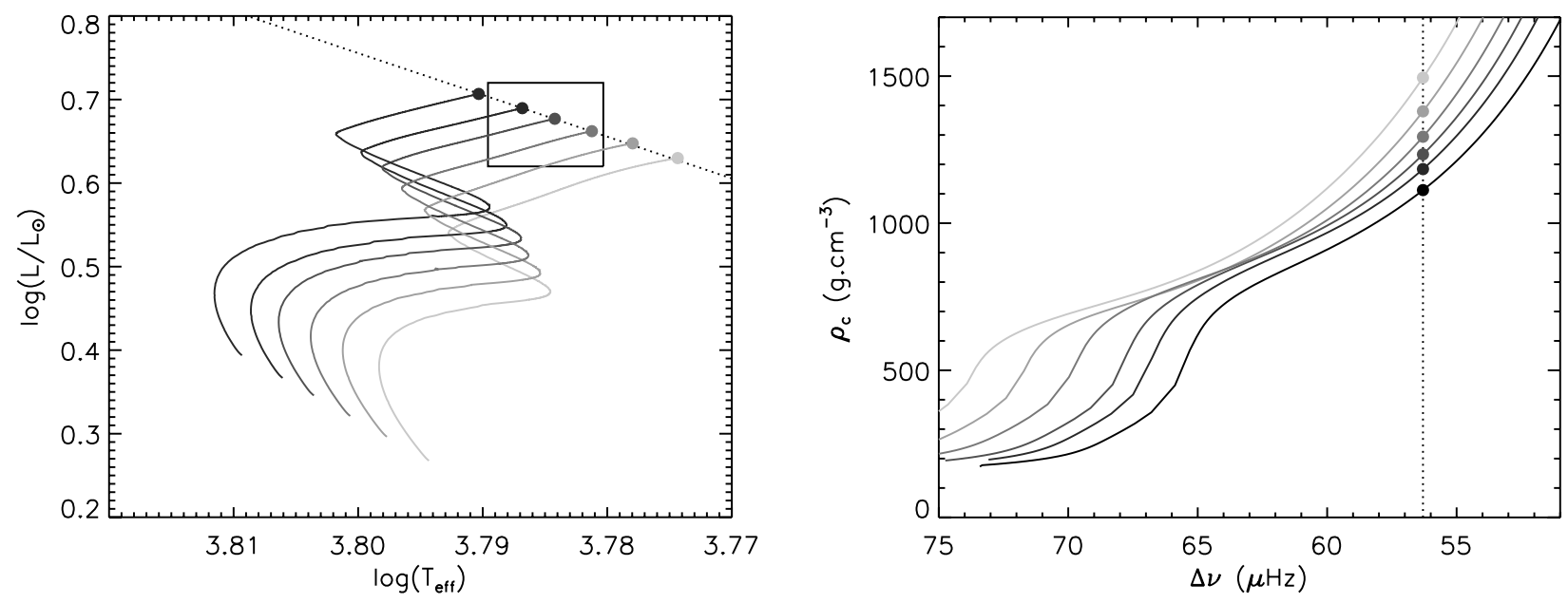

Fig. 11. Left: evolutionary tracks in the HR diagram for models of masses ranging from $1.23 M_{\odot}$ (lightest symbols) to $1.31 M_{\odot}$ (darkest symbols), with given physics. The filled circles indicate the points where the models reach $\langle\Delta v\rangle=\langle\Delta v\rangle_{\text {obs }}$ for HD 49385 (this location is materialized by the dotted line). Right: evolution of the central density for the same models. The value of the mean large separation has been used as an indicator of the age, for more convenience.

parameter of the fit. This is very interesting because it solves the problem of the choice of a time step for the grid of models.

We note that by using this method, we overlook the models that do not fit the frequency of the avoided crossing within $1-\sigma$ error bars. However, since avoided crossings occur on a short timescale relative to the stellar evolution timescale, modifying $v_{\text {cross }}$ in a model will result in almost no change to the other stellar parameters. It will only induce an increase in the contribution of the term including $v_{\text {cross }}$ in the $\chi^{2}$ defined in Eq. (2) with no significant change in the other contributions. Overlooking these models should therefore change neither the optimal model we obtain nor the error bars we derive from it.

\subsubsection{Correspondence between ( $\left.\Delta v, v_{\text {cross }}\right)$ and ( $M$, age)}

We have just demonstrated that for a model of a given mass and physics, we can find an age such that $v_{\text {cross }}=v_{\text {cross }}^{\text {obs }}$. However, we note that the mean large separation of the star monotonically decreases with age because the stellar radius keeps increasing (even during the sharp transition between the main-sequence and the post-main-sequence stage). For every model, there exists an age at which the observed value of the mean large separation is reproduced. This age a priori differs from the one for which the frequency of the avoided crossing is reproduced. Hence, which models fit both conditions simultaneously? We refer to this joint condition as condition $C$, i.e.

$C:\left\{\begin{array}{l}\langle\Delta v\rangle_{\bmod }=\langle\Delta v\rangle_{\mathrm{obs}} \\ v_{\text {cross }}^{\bmod }=v_{\text {cross }}^{\text {obs }} .\end{array}\right.$

To answer this question, we search for models that fit the observed frequency of the avoided crossing among those that reproduce the value of the mean large separation. As explained in Appendix A, the iso- $\Delta v$ region in the HR diagram roughly corresponds to a line of slope $5\left(L \propto T_{\text {eff }}^{5}\right)$. Figure 11 shows the evolutionary tracks of several models with the same given physics and different masses. We observe that as the stellar mass increases, the line of the terminal age main sequence (TAMS) gets closer to the line of iso- $\Delta v$. Consequently, the more massive stars are closer to the TAMS when they reach the observed value of the large separation. This means that they have a lower central density, as can be seen in Fig. 11 (where we found it convenient to

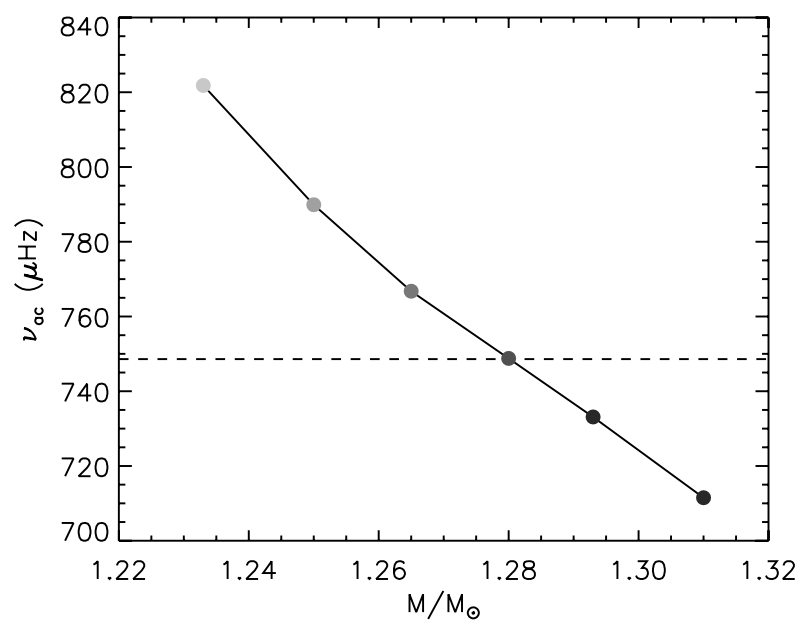

Fig. 12. Frequency of the avoided crossing for the models that reproduce the observed mean large separation of HD 49385 in Fig. 11. The dashed line indicates the frequency of the observed avoided crossing $v_{\text {cross }}^{\text {obs }}$ for HD 49385.

use the mean large separation instead of the age as an indicator of the evolution). Since we mentioned that the evolution of $v_{\text {cross }}$ is determined by that of $\rho_{\mathrm{c}}$, we conclude that $\nu_{\text {cross }}$ should decrease with increasing mass. This is confirmed by Fig. 12. We refer to this result frequently in the following.

We reach the conclusion that there exists one and only one value of the stellar mass and age for which both the mean value of the large separation and the frequency of the avoided crossing are correctly fitted (for instance with the physics used in Figs. 11 and 12 , it corresponds to a mass of about $1.28 M_{\odot}$ ). We denote as $\tilde{M}$ and $\tilde{\tau}$ the stellar mass and age that satisfy condition $C$. It is striking that for a given physics, the two seismic parameters $\langle\Delta v\rangle$ and $v_{\text {cross }}$ alone provide an estimate of the stellar mass and age, without having to resort to the use of any classical constraints or other seismic parameters. This estimate is also extremely precise. For example, if we fix all stellar parameters other than the mass and age in the case shown in Fig. 12, the uncertainty in he mass that we obtain is as small as $4 \times 10^{-4} M_{\odot}$. We therefore propose to reduce the dimensions of the model space by eliminating the mass and age from the set of free parameters, 
these two quantities being determined by $\left(\langle\Delta v\rangle, v_{\text {cross }}\right)$. The values of $\tilde{M}$ and $\tilde{\tau}$ of course depend on the physics that we use to model the star. Their dependence on the different stellar parameters is studied in Sect. 4.5, after we describe the method we adopted in this study to find the models that satisfy condition $C$.

\subsection{Searching for models satisfying condition $C$}

We now briefly describe the procedure we followed to obtain the values of $\tilde{M}$ and $\tilde{\tau}$ for each set of free parameters.

As mentioned in Sect. 4.3.1, we consider the stellar age to be fixed by imposing that $v_{\text {cross }}=v_{\text {cross }}^{\text {obs }}$. Until now, we have assumed that the frequency of the avoided crossing corresponds to the frequency of the uncoupled g mode that it involves. Of course, when computing the eigenfrequencies of a model, we do not have access to this quantity. We thus chose to assimilate the frequency of the avoided crossing to the frequency of the mode that behaves mainly like a g mode. In the oscillation spectrum of HD 49385, this mode corresponds to the peak labeled as $\pi_{1}$, whose frequency has been found to be $v_{\pi_{1}}=748.6 \pm$ $0.23 \mu \mathrm{Hz}$ by D10, and in the models, it is the mode $v_{1,11}$. For each computed model, the age is determined by ensuring that $v_{1,11}=v_{\pi_{1}}$. Special care has to be taken in this procedure since the evolution of the $\ell=1$ mode frequencies with age is not monotonic owing to the avoided crossings, as can clearly be seen in Fig. 10. As a result, there are several ages (three) for which $\left(v_{1,11}=v_{\pi_{1}}\right)$ and only one among them corresponds to the one we search for.

We need to determine the stellar mass for which the models fitting the frequency of the avoided crossing also reproduce the observed large separation. This mass is the one that minimizes the merit function

$\chi_{\langle\Delta v\rangle}^{2} \equiv \frac{\left(\langle\Delta v\rangle_{\mathrm{mod}}-\langle\Delta v\rangle_{\mathrm{obs}}\right)^{2}}{\sigma_{\langle\Delta v\rangle}^{2}}$,

where $\langle\Delta v\rangle_{\text {obs }}$ is the mean large separation of the observed radial modes, $\sigma_{\langle\Delta v\rangle}$ the 1- $\sigma$ error-bar, and $\langle\Delta v\rangle_{\bmod }$ the corresponding quantity for the models (after correcting for the surface effects as described in Sect. 2.2.2). Only the radial modes are considered here since the large separations of non-radial modes are affected by avoided crossings. We note that for models reproducing $v_{\text {cross }}^{\text {obs }}$, the mean large separation of radial modes varies almost linearly with stellar mass. The function $\chi_{\langle\Delta v\rangle}^{2}$ is therefore almost quadratic and the method of Newton is particularly efficient in determining the optimal mass $\tilde{M}$ (obtained in only a couple of iterations).

\subsection{Dependence of $\tilde{M}$ on the stellar parameters}

By applying the procedure we have just described, the mass $\tilde{M}$ and age $\tilde{\tau}$ can be determined for any given set of free parameters. This gives us the opportunity to study the dependence of $\tilde{M}$ on the different stellar parameters that are varied in this study (the mixing length parameter $\alpha_{\text {conv }}$, the initial helium abundance $Y_{0}$, the metallicity $[Z / X]$, and the amount of core overshooting $\alpha_{\mathrm{ov}}$ ). This provides us with helpful insights when discussing the results of our optimization in Sect. 6. This should also give some insight into how the mass should be varied when studying other stars with avoided crossings.

We start from a model $S_{0}$ with a given set of stellar parameters (see Table 2). From what we have just said, there exists only one stellar mass $\tilde{M}_{0}$ and and one age $\tilde{\tau}_{0}$ for which condition $C$ is verified, for these parameters. By varying the stellar parameters one by one, we try to understand the variations in the optimal mass $\tilde{M}$ with each of them.

\subsubsection{Dependence of $\tilde{M}$ on the mixing length parameter}

We modified the mixing length parameter of model $S_{0}$ without changing the other parameters and we determined the new mass $\tilde{M}$ by applying the procedure described in Sect. 4.4. Figure 13a shows that $\tilde{M}$ linearly increases with $\alpha_{\text {conv }}$.

The increase in $\tilde{M}$ with $\alpha_{\text {conv }}$ can be understood as follows. We first modify the mixing length parameter from the value $\alpha_{0}$ of model $S_{0}$ to a value $\alpha_{1}>\alpha_{0}$. If we keep the same mass $\tilde{M}_{0}$ (model $S_{1 \mathrm{a}}$ in Table 2), an increase in the mixing length will lead to an increase in the convective flux in the envelope and therefore cause the star to contract slightly. Since the stellar luminosity remains unchanged, the decrease in the radius is compensated for by an increase in the effective temperature. The evolutionary track is thus horizontally translated to the left in the HR diagram when the mixing length goes from $\alpha_{0}$ to $\alpha_{1}$ at constant mass (see Fig. 13b). As a result, for $\alpha_{\text {conv }}=\alpha_{1}$, the star is further away from the TAMS when it reaches the observed value of the large separation and the frequency of the avoided crossing is too high relative to the observations. On the basis of our discussion in Sect. 4.3.2, we need to increase the stellar mass to restore the agreement with the avoided crossing frequency, while maintaining the agreement with the large separation (model $S_{1 \mathrm{~b}}$ ). We therefore have $\tilde{M}_{1}>\tilde{M}_{0}$.

\subsubsection{Dependence of $\tilde{\mathrm{M}}$ on the helium abundance}

By modifying the value of the initial helium abundance in model $S_{0}$, we show that $\tilde{M}$ linearly decreases with $Y_{0}$ (see Fig. 13c).

As in the previous section, we try to explain this decrease. We search for the new mass $\tilde{M}_{2}$ that verifies condition $C$ when we increase the helium abundance to $Y_{2}>Y_{0}$. If we keep the same mass $\tilde{M}_{0}$ (model $S_{2 \mathrm{a}}$ ), the mean molecular weight $\mu$ increases owing to the increase in the helium content. It can be shown from homology relations that if we keep the mass constant and modify $\mu$, the stellar luminosity scales as $L \propto \mu^{4}$ and the radius scales as $R \propto \mu^{z_{2}}$, where the exponent $z_{2}$ depends on the mode of energy generation inside the star (see e.g., Kippenhahn \& Weigert 1990). Subgiants are generally burning hydrogen through the CNO cycle and we then have $z_{2} \simeq 0.6$. In this case, combining these two relations with the knowledge that $L \propto R^{2} T_{\text {eff }}^{4}$, we find that $T_{\text {eff }} \propto L^{5.7}$. Increasing the helium abundance in a model while keeping the mass constant induces a translation of its evolutionary track in the HR diagram to the left along a line of slope about 5.7. Since the slope is steeper than that of the iso- $\Delta v$ line, the models with higher helium abundance are closer to the TAMS when they reach $\Delta v_{\text {obs }}$ (green dashed line in Fig. 13d). This effect is enhanced by the finding that when increasing $Y_{0}$, the iso- $\Delta v$ line is translated to the left. To reproduce the observed value of $v_{\text {cross }}$, it is therefore necessary to decrease the mass, which means that $\tilde{M}_{2}<\tilde{M}_{0}\left(\operatorname{model} S_{2 \mathrm{~b}}\right)$.

\subsubsection{Dependence of $\tilde{M}$ on the metallicity}

Figure $13 \mathrm{e}$ shows that the mass $\tilde{M}$ linearly increases with the abundance of heavy elements $[Z / X]$.

To explain this increase, we consider a metallicity $[Z / X]_{3}>$ $[Z / X]_{0}$ and search for the optimal mass $\tilde{M}_{3}$. If we keep the mass $\tilde{M}_{0}$ of model $S_{0}$ (model $S_{3 \mathrm{a}}$ ), the main effect of an increase 

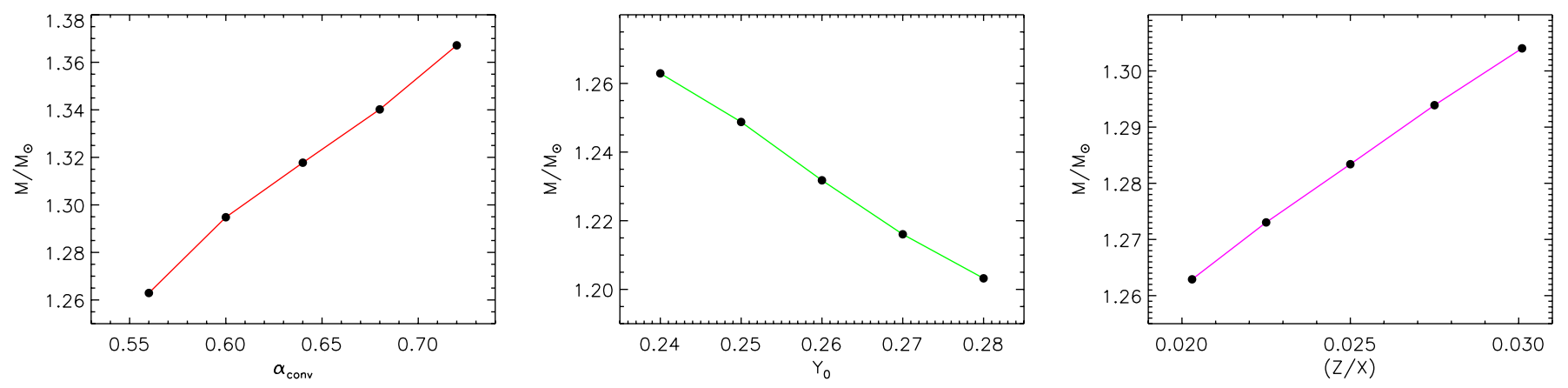

Fig. 13. Variations in the mass $\tilde{M}$ when changing the mixing length parameter (left), the initial helium abundance (middle), and the metallicity (right) from model $S_{0}$ (whose properties are given in Table 2).

Table 2. Parameters of the models used to determine the variations in the mass $\tilde{M}$ (which verifies condition $C$ ) with the stellar parameters.

\begin{tabular}{lcccc}
\hline \hline Model & $\alpha_{\text {conv }}$ & $Y_{0}$ & Mixture & Mass \\
\hline$S_{0}$ & 0.56 & 0.24 & AGS05 & $\tilde{M}_{0}\left(1.263 M_{\odot}\right)$ \\
\hline$S_{1 \mathrm{a}}$ & 0.60 & - & - & $\tilde{M}_{0}$ \\
$S_{1 \mathrm{~b}}$ & 0.60 & - & - & $\tilde{M}_{1}\left(1.295 M_{\odot}\right)$ \\
\hline$S_{2 \mathrm{a}}$ & - & 0.28 & - & $\bar{M}_{0}$ \\
$S_{2 \mathrm{~b}}$ & - & 0.28 & - & $\tilde{M}_{2}\left(1.202 M_{\odot}\right)$ \\
\hline$S_{3 \mathrm{a}}$ & - & - & GN93 & $\tilde{M}_{0}$ \\
$S_{3 \mathrm{~b}}$ & - & - & GN93 & $\tilde{M}_{3}\left(1.308 M_{\odot}\right)$ \\
\hline
\end{tabular}

in the metallicity is that the amount of bound-free absorption, which comes from metals, is higher. This leads to an increase in the opacity. To study its effect, we computed homology relations, following exactly the same steps as in Chap. 20 of Kippenhahn $\&$ Weigert (1990), but considering the opacity $\kappa$ as a basic parameter instead of the mean molecular weight $\mu$. We found that if we keep the mass constant while increasing the opacity, the evolutionary track in the HR diagram is translated to the right on a line of slope about 3.6 (we note that the models indeed show a translation to the right, but with a somewhat larger slope a little below 5, see purple dashed line in Fig. 13f). Normally, this should bring the models closer to the TAMS when they reach the observed value of the large separation. However, we observe in Fig. 13 that the iso- $\Delta v$ line is shifted to the right when increasing the opacity and the models with higher metallicity are further away from the TAMS (purple full line in Fig. 13f). We therefore need to increase the mass so that the frequency of the avoided crossing is correctly reproduced, i.e. $\tilde{M}_{3}>\tilde{M}_{0}\left(\operatorname{model} S_{3 \mathrm{~b}}\right)$.

\subsubsection{Dependence of $\tilde{\mathrm{M}}$ on the amount of overshooting}

We now focus on the influence of the overshooting on the mass $\tilde{M}$, which is more complex than the influence of the stellar parameters previously studied. Starting from model $S_{0}$, we increased the amount of overshooting and determined the mass $\tilde{M}$. Figure 16 shows that $\tilde{M}$ increases with $\alpha_{\text {ov }}$ for low overshooting coefficients $\left(\alpha_{\text {ov }}<0.06\right)$. Above this limit, the mass $\tilde{M}$ starts to decrease as a function of $\alpha_{\mathrm{ov}}$. This decrease eventually becomes linear for $\alpha_{\text {ov }}>0.1$. We note that the shape of $\tilde{M}\left(\alpha_{\text {ov }}\right)$ does not qualitatively change if we consider initial models other than $S_{0}$ (even though the value of the transitional $\alpha_{\mathrm{ov}}$ does vary).

Core overshooting is not supposed to play a direct role because the models we consider are in the PoMS stage and their convective core has vanished. However, it does have an impact on the g-mode frequencies because of its influence on the past

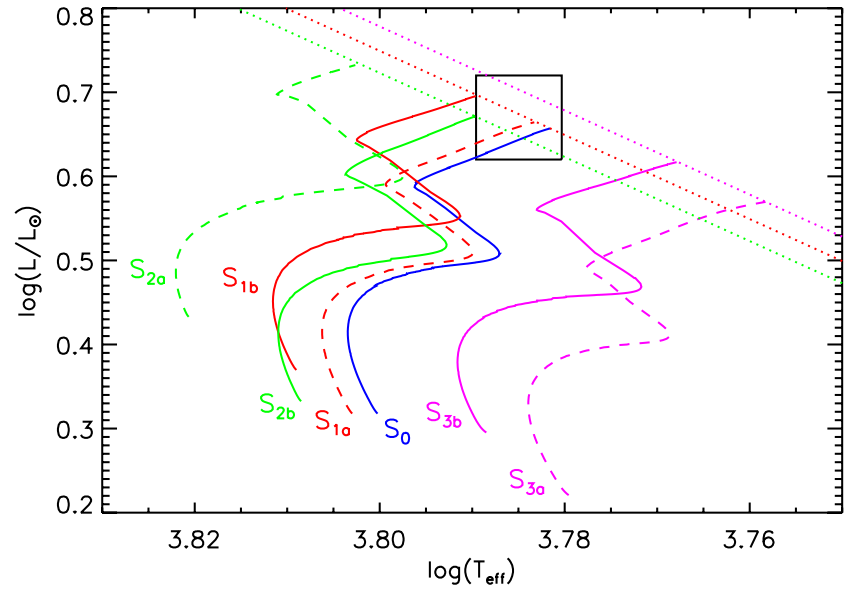

Fig. 14. Evolutionary tracks in the HR diagram of models $S_{0}, S_{1 \mathrm{a}}, S_{1 \mathrm{~b}}$, $S_{2 \mathrm{a}}, S_{2 \mathrm{~b}}, S_{3 \mathrm{a}}$, and $S_{3 \mathrm{~b}}$ (see text and Table 2 ).

evolution of the star and the chemical composition at the center. Adding overshooting has two opposing effects on the frequency of the avoided crossing:

(i) First, by extending the mixed region associated with the convective core, overshooting increases the size of the hydrogen reservoir available for the nuclear reactions in the center. As a result, the star remains longer in the MS stage, as clearly appears in Fig. 15. Consequently, for a given fixed mass, an increase in the amount of overshooting brings the star closer to the TAMS when it reaches the observed value of the large separation. On the basis of our discussion in Sect. 4.3.2, we expect the frequency of the avoided crossing to decrease when the amount of overshooting increases.

(ii) However, adding overshooting also increases the size of the mixed core during the MS stage and therefore, when it reaches the TAMS, its helium core is bigger. The layers where hydrogen remains in the star are less deep and the temperature in these regions is thus lower. The contraction of the star during the PoMS stage needs to be stronger to trigger nuclear burning in these shells. As a result, the central density increases faster with overshooting than it does without overshooting, as can be seen in Fig. 15. Since the frequency of the avoided crossing is strongly related to the central density (see Sect. 4.3.1), we expect $\nu_{\text {cross }}$ to grow whenever $\alpha_{\mathrm{ov}}$ increases.

These two effects play different roles, depending on the amount of overshooting. For mild overshooting, the stars have evolved sufficiently after the TAMS when they reach $\langle\Delta v\rangle_{\text {obs }}$ to ensure 

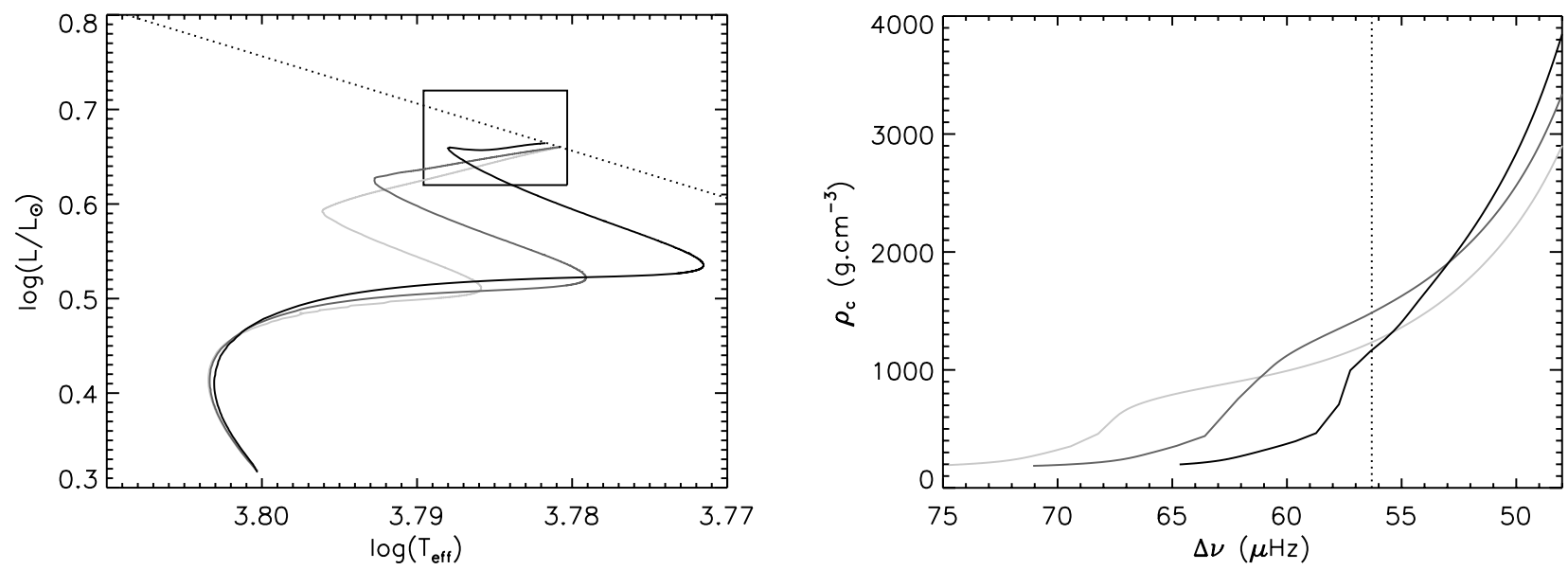

Fig. 15. Evolutionary tracks in the HR diagram (left) and evolution of the central density $\rho_{\mathrm{c}}$ (right) for models with the same mass but different values of $\alpha_{\mathrm{ov}}\left(\alpha_{\mathrm{ov}}=0,0.08\right.$ and 0.15 from light gray to dark gray). The observed mean value of the large separation is represented by the dotted line.

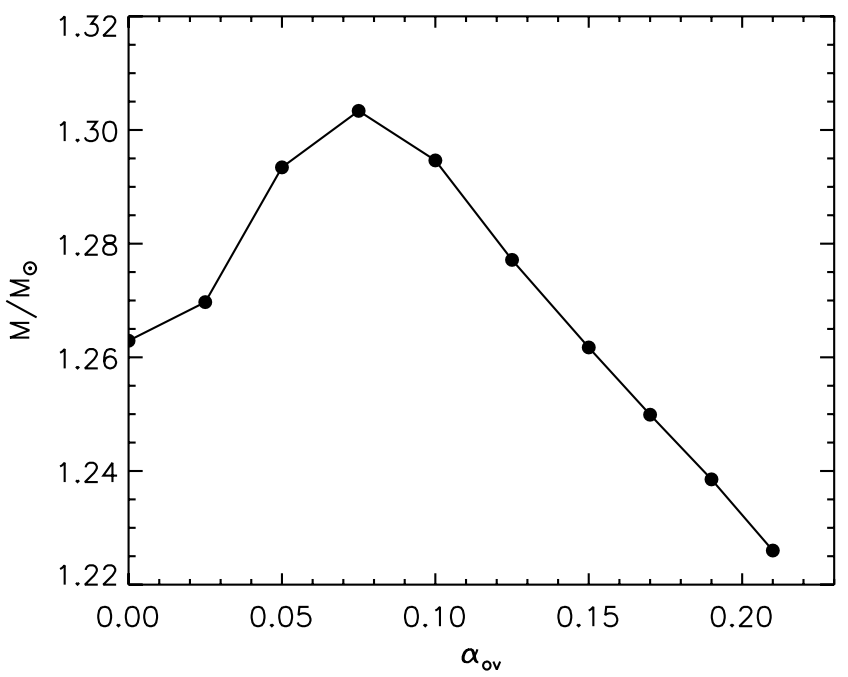

Fig. 16. Variations in the optimal mass $\tilde{M}$ with the overshooting coefficient $\alpha_{\text {ov }}$. Two regimes can be distinguished: for a strong overshooting, the models are close to the TAMS and effect (i) dominates $\left(\alpha_{\mathrm{ov}}>0.1\right)$ whereas for a milder amount of overshooting, effect (ii) takes over $\left(\alpha_{\mathrm{ov}}<0.1\right)$.

that the second effect prevails (see Fig. 15). When increasing the amount of overshooting of model $S_{0}$ from $\alpha_{\mathrm{ov}}=0$ to $\alpha_{\mathrm{ov}}=0.08$, $v_{\text {cross }}$ increases, which means that the stellar mass needs to be increased to ensure that condition $C$ is satisfied. This accounts for the increase in $\tilde{M}$ at low $\alpha_{\text {ov }}$.

On the other hand, for larger amounts of overshooting, the stars are very close to the TAMS when they reach $\langle\Delta v\rangle_{\text {obs. }}$. The central density has not had time to increase sufficiently and the first effect is dominant. Figure 15 indeed shows that if we increase the overshooting to $\alpha_{\mathrm{ov}}=0.15$, the central density decreases, which means that the frequency of the avoided crossing also decreases. Therefore, the mass needs to be decreased to verify condition $C$.

We note that owing to the competition between these two effects, for certain masses two different models can both fulfill condition $C$ : one with a mild overshooting away from the TAMS, and the other with a larger $\alpha_{\text {ov }}$, close to the TAMS. For instance for the physics chosen in Fig. 16, a mass of $M=1.263 M_{\odot}$
Table 3. Ranges and steps adopted for the free parameters of the computed grids of models.

\begin{tabular}{lcc}
\hline \hline Parameters & Range & Step \\
\hline$Y_{0}$ & 0.24 to 0.28 & 0.01 \\
$\alpha_{\text {conv }}$ & 0.48 to 0.72 & 0.04 \\
{$[Z / X]($ dex $)$} & 0.04 to 0.14 & 0.05 \\
$\alpha_{\text {ov }}$ & 0.00 to 0.20 & 0.025 \\
\hline
\end{tabular}

can satisfy condition $C$ both without overshooting $\left(\alpha_{\mathrm{ov}}=0\right)$ and with an overshooting of $\alpha_{\mathrm{ov}}=0.15$. This observation shows that for subgiants in the early PoMS stage, knowing the frequency of an avoided crossing is not enough to efficiently constrain the amount of core overshooting in a star.

\section{Application to HD 49385: model optimization}

\subsection{Computation of two grids of models}

We computed two grids of models, one assuming the mixture of Grevesse \& Noels (1993, furhter noted GN93) and the other the more recent mixture of Asplund et al. (2005, further noted AGS05). We varied the values of the mixing length parameter $\alpha_{\text {conv }}$, the helium abundance $Y_{0}$, the metallicity $[Z / X]$ and the amount of overshooting $\alpha_{\mathrm{ov}}$. The ranges of studied values for each of these parameters as well as the chosen steps are reported in Table 3. For each point in the grids, an optimization such as the one described in Sect. 4.4 was performed to determine the stellar mass $\tilde{M}$ and age $\tilde{\tau}$ that satisfy condition $C$. We then computed the $\chi^{2}$ function defined by Eq. (2).

\subsection{Results of the fit}

The first comment that can be made about the fit is that the representation of the total $\chi^{2}$ as a function of the amount of overshooting shown in Fig. 17 has two distinct minima, one for lower values of $\alpha_{\mathrm{ov}}\left(\alpha_{\mathrm{ov}}<0.05\right)$ and the other for moderate values of $\alpha_{\text {ov }}(\sim 0.19)$. This leads us to identify four different families of solutions corresponding to the two different mixtures we considered and either low or high values of $\alpha_{\mathrm{ov}}$.

We then attempted to derive a quantitative interpretation of the $\chi^{2}$ values we obtain for the models in each of these four 
Table 4. Values of the stellar parameters obtained from the computation of our two grids of models.

\begin{tabular}{lcccc}
\hline \hline & \multicolumn{2}{c}{ GN93 } & \multicolumn{2}{c}{ AGS05 } \\
\hline$\alpha_{\text {ov }}$ & low $\alpha_{\text {ov }}$ & high $\alpha_{\text {ov }}$ & low $\alpha_{\text {ov }}$ & high $\alpha_{\text {ov }}$ \\
\hline$M / M_{\odot}$ & $0.05_{-0.05}^{+0.01}$ & $0.19 \pm 0.01$ & $0.00^{+0.01}$ & $0.19 \pm 0.01$ \\
Age $(\mathrm{Gyr})$ & $1.285 \pm 0.017$ & $1.231 \pm 0.012$ & $1.264 \pm 0.013$ & $1.210 \pm 0.021$ \\
$R / R_{\odot}$ & $4.90 \pm 0.13$ & $5.04 \pm 0.18$ & $4.88 \pm 0.11$ & $5.10 \pm 0.18$ \\
$T_{\text {eff }}(\mathrm{K})$ & $1.959 \pm 0.012$ & $1.932 \pm 0.011$ & $1.947 \pm 0.007$ & $1.917 \pm 0.011$ \\
$\log g$ & $5870 \pm 40$ & $5790 \pm 30$ & $5940 \pm 40$ & $6080 \pm 60$ \\
$\alpha_{\text {conv }}$ & $3.961 \pm 0.002$ & $3.946 \pm 0.001$ & $3.960 \pm 0.002$ & $3.954 \pm 0.002$ \\
\hline Minimum $\chi_{\text {red }}^{2}$ & $0.52 \pm 0.01$ & $0.52 \pm 0.02$ & $0.52 \pm 0.01$ & $0.56 \pm 0.03$ \\
\hline
\end{tabular}

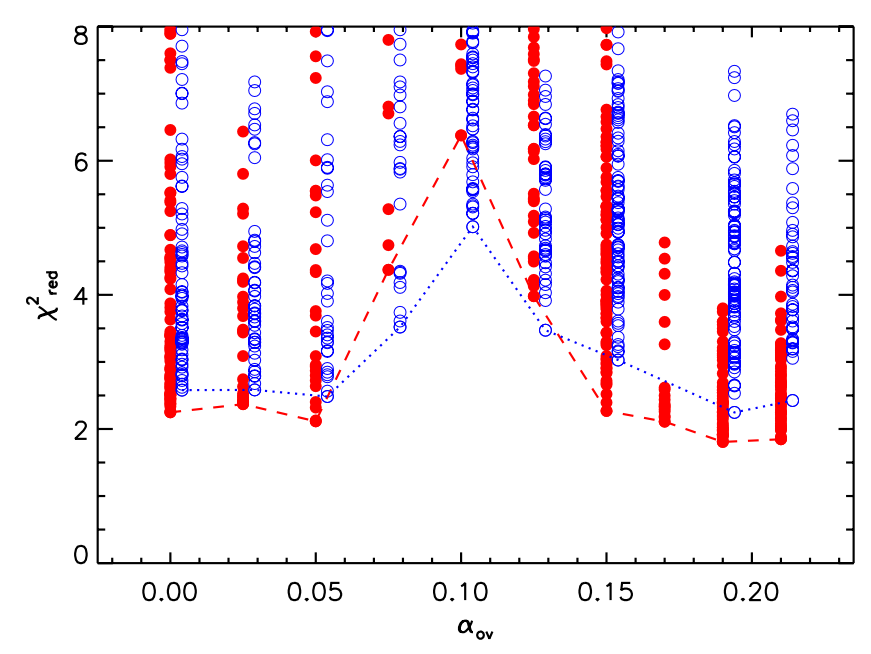

Fig. 17. Values of the reduced $\chi^{2}$ for the models computed with the mixture of GN93 (blue empty circles) and with the mixture of AGS05 (red filled circles) as a function of the amount of overshooting. For clarity reasons, the symbols of models computed with GN93 have been slightly shifted to the right. The best-fit models for each considered value of $\alpha_{\mathrm{ov}}$ have been linked by a dotted line (GN93) and by a dashed line (AGS05).

families. This was possible because (i) the measured data can be assumed to have a Gaussian distribution (Appourchaux et al. 1998 , showed that for simulated spectra with a resolution equivalent to four months of data, the distribution of the error bars for the central frequencies of the modes is close to a normal distribution) and (ii) for the four families of solutions, the models are roughly linear in their parameters in the regions defined by the uncertainties that we derive for the fitted parameters. This latter condition has been checked a posteriori.

Under these conditions, the goodness of the fit can be estimated by computing the reduced $\chi^{2}$ defined as

$\chi_{\text {red }}^{2} \equiv \frac{\chi^{2}}{N-P}$

where $N$ is the number of measured data and $P$ the number of free parameters of the fit. More importantly, we can use the values of the $\chi^{2}$ function to determine a confidence interval for the fitted parameters. For instance, let us assume that we wish to determine the uncertainty in the amount of overshooting $\alpha_{\mathrm{ov}}$. For each value of $\alpha_{\text {ov }}$ in the grid, we determine the set of the other free parameters that minimizes the $\chi^{2}$ function (the obtained $\chi^{2}$ values are linked by dotted lines in Fig. 17). Press et al. (2002) showed that the difference between these $\chi^{2}$ values and the minimum $\chi^{2}$ of the whole family of solutions $\chi_{\min }^{2}$ defined as

$\Delta \chi^{2} \equiv \chi^{2}-\chi_{\min }^{2}$

is distributed as a $\chi^{2}$ with two degrees of freedom. This means that the "real" value of the parameter $\alpha_{\text {ov }}$ has a probability of $68.3 \%$ (resp. $95.4 \%, 99.7 \%$ ) of belonging to the region where $\Delta \chi^{2}<1$ (resp. $\Delta \chi^{2}<4, \Delta \chi^{2}<9$ ). The values of $\alpha_{\text {ov }}$ for which $\Delta \chi^{2}=1$ thus give an estimate of the $1-\sigma$ error in this parameter. This method is applied to all the parameters to determine the uncertainties that are reported in Table 4 for the four different families of solutions.

We thus obtain a precise estimate of the fundamental properties of HD 49385. The stellar mass is constrained with a precision of about $1 \%$ for each of the four identified families. If we combine them all together, we find a mass of $M=1.25 \pm 0.05 M_{\odot}$ for HD 49385 (corresponding to a precision of about $4 \%$ ). In a similar way, we obtain a radius of $R=1.94 \pm 0.03 R_{\odot}$ for the star (precision of about $1.5 \%$ ). The stellar age is also tightly constrained: from Table 4 , we derive $\tau=5.02 \pm 0.26 \mathrm{Myr}$ (precision of about $5 \%$ ). We obtain a $\log g$ of $3.954 \pm 0.009$ for HD 49385. We note that the results of the fit are consistent with the first estimates given in Table 1. The level of precision that we achieve here is to a great extent due to the detection of the $\ell=1$ avoided crossing in the spectrum of HD 49385 (see Sect. 6).

As expected, we find that we are also able to obtain information about the interior of HD 49385. As we mentioned at the beginning of this section, we obtain strong constraints on the amount of overshooting in the star that can be either very low or moderate $\left(\alpha_{\mathrm{ov}}=0.19 \pm 0.01\right)$. In the latter case, the models are very close to the TAMS. We know that stars are expected to spend very little time in this evolutionary stage, which makes it quite improbable that we can observe them during this period. Although this point in itself does not justify our ignoring these solutions, it means that we should treat them with caution. The best-fit models of the two families we obtained share very similar values of all the observables available for HD 49385, as shown in Fig. 18, so that we cannot discriminate between them. We note however that the oscillation spectra of the models contain an $\ell=2$ mixed mode in the frequency range of the observations, which has different frequencies for the bestfit low-overshooting models (between 973 and $984 \mu \mathrm{Hz}$ ) and for the high-overshooting ones (between 998 and $1002 \mu \mathrm{Hz}$ ). If such a mode could be detected in the observed spectrum, we 

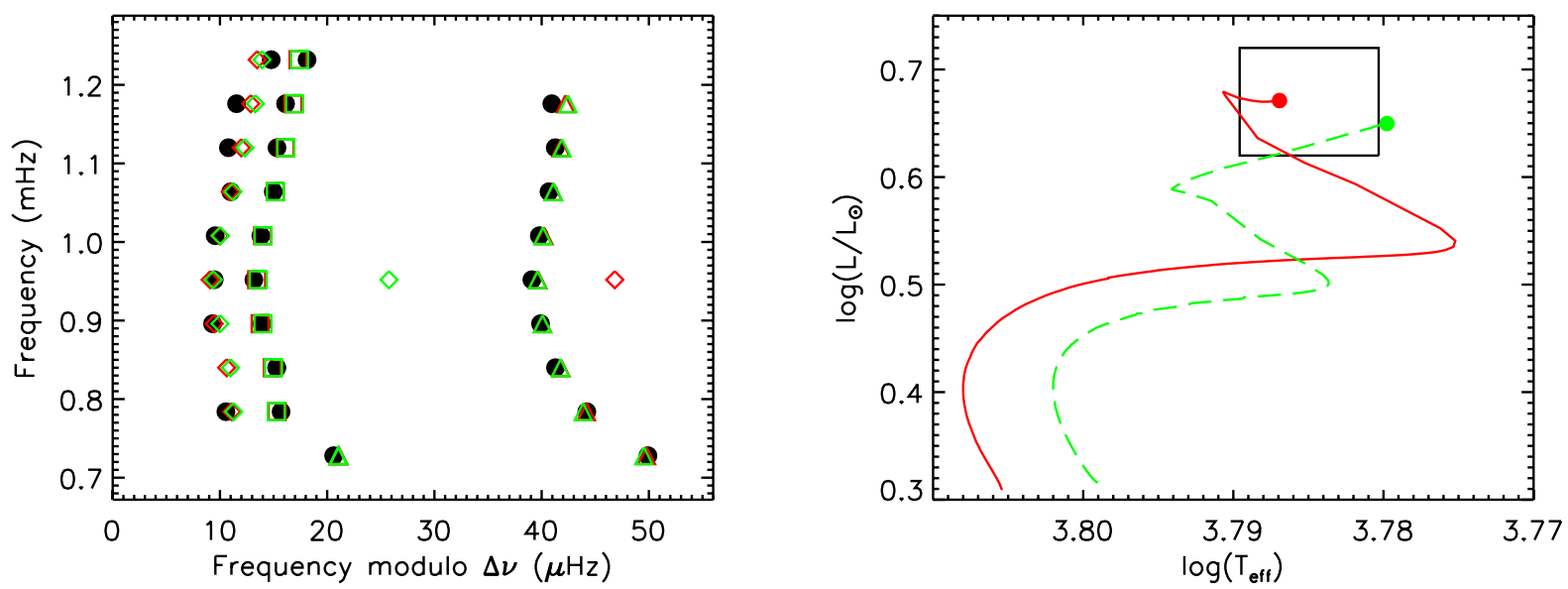

Fig. 18. Left: échelle diagrams of the best-fit models with low overshooting (green) and with high overshooting (red). The squares represent $\ell=0$ modes, triangles $\ell=1$ modes, and diamonds $\ell=2$ modes. The observations are represented by the black filled circles. Right: evolutionary tracks in the HR diagram of the two models (green dashed line: low overshooting; red solid line: high overshooting).

would have a way of discriminating between the two scenarios. For completeness sake, we checked how the frequencies of the peaks $\pi_{2}$ and $\pi_{3}$ of D10 compare with the $\ell=3$ frequencies in our models. In both cases (low and moderate $\alpha_{\mathrm{ov}}$ ), $\pi_{2}$ and $\pi_{3}$ appear between 2 and $3 \mu \mathrm{Hz}$ higher than the theoretical values (i.e. between 3 and $5 \sigma$ ).

An interesting feature of the fit is that the mixing length parameter $\alpha_{\text {conv }}$ is well-constrained for HD 49385 and corresponds to a value that is significantly smaller than the solar one, regardless of the family of solution. Combining the families together, we obtain $\alpha_{\text {conv }}=0.55 \pm 0.04$ (we recall that the solar calibrated value is $\left.\alpha_{\text {conv }}=0.64\right)$. To strengthen this result, we checked that we reached similar conclusions when using the traditional mixing length theory instead of the formalism of Canuto et al. (1996). In this case, the closest agreement is reached for a mixing length of $\alpha_{\mathrm{MLT}} \sim 1.4$, which is significantly smaller than the solar calibrated value $\alpha_{\text {MLT }} \sim 1.85$.

We also find that the models computed with the abundances of heavy elements of AGS05 offer a closer agreement with the observations than the ones computed with the abundances of GN93. This can be clearly seen in Fig. 19. This result is strengthened by the fact that even with the mixture of GN93, the best-fit models are found when considering the lowest end of the range of the tested metallicities, i.e. $[Z / X]=0.04$ dex.

These results confirm that the seismic constraints available for HD 49385 provide valuable information about the interior of the star. However, they raise a certain number of questions which the grid-of-model approach in itself cannot answer. Why do we find two distinct families of solutions depending on the value of $\alpha_{\mathrm{ov}}$ ? How do we manage to constrain the mixing length parameter $\alpha_{\text {conv }}$ so well in this star? Why do lower metallicities provide tighter fits than higher ones? Of what use are the information conveyed by the $\ell=1$ ridge distortion about the coupling between the cavities? We try to address these questions in the following section.

\section{An in-depth analysis of the results of the fit}

\subsection{Sources of contribution to the $\chi^{2}$ function}

For the models computed in Sect. 5, we identified three main sources of contribution to the value of the $\chi^{2}$ function.

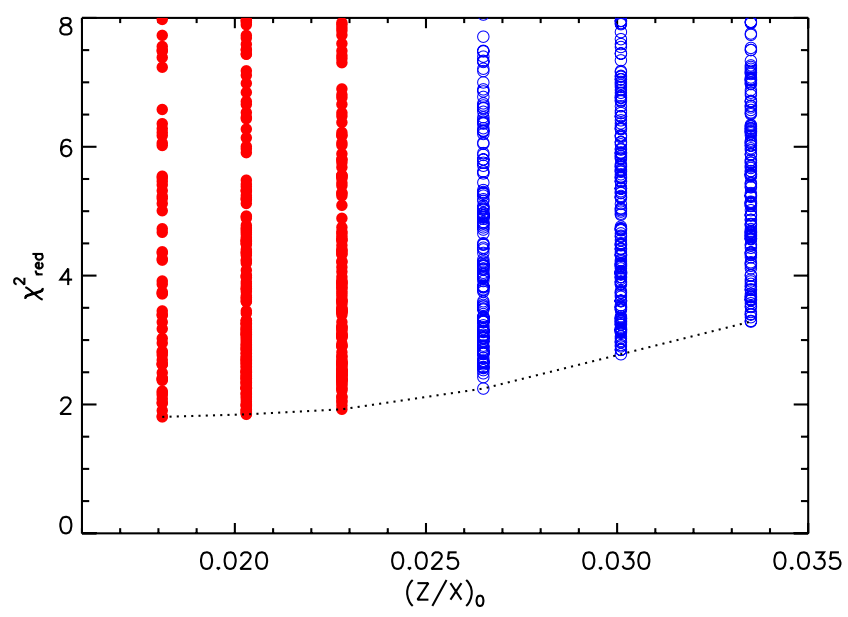

Fig. 19. Values of the reduced $\chi^{2}$ as a function of metallicity $[Z / X]$. Symbols are the same as in Fig. 17.

The position in the HR diagram. For each point of the grid, the mass and age are fixed to $\tilde{M}$ and $\tilde{\tau}$, which determines a certain position in the HR diagram. With the abundances of AGS05, the values of $T_{\text {eff }}$ and $L$ contribute only weakly to the $\chi^{2}$ of the bestfit models (it represents only $0.3 \%$ of the total $\chi^{2}$ for our best-fit model). The overall fit is thus fully consistent with the spectroscopic constraints. With the abundances of GN93, the fits point toward an effective temperature that is about 3- $\sigma$ lower than the measured one (see Table 4), which results in a significant contribution to the $\chi^{2}$ value (more than $20 \%$ of the total $\chi^{2}$ for the best-fit models).

The oscillation of the frequencies due to the $\Gamma_{1}$ bump. We have seen in Sect. 2.1.2 that a clear oscillation is observed in the large separation profiles. The models also show an oscillation with a comparable period, which is caused by the bump of $\Gamma_{1}$ in the helium second ionization zone (HIZ) at an acoustic depth of the order of $\tau_{\mathrm{HIZ}} \sim 0.8$. We note that another oscillation related to the base of the convection zone (BCZ) should also be present with a period of around $120 \mu \mathrm{Hz}\left(\tau_{\mathrm{BCZ}} \sim 0.5\right)$. However, its amplitude is too low to be detected. The closest agreement with the observed oscillation is obtained for $\tau_{\mathrm{HIZ}} \sim 0.798$, which is consistent with the estimate of the acoustic depth of the glitch 


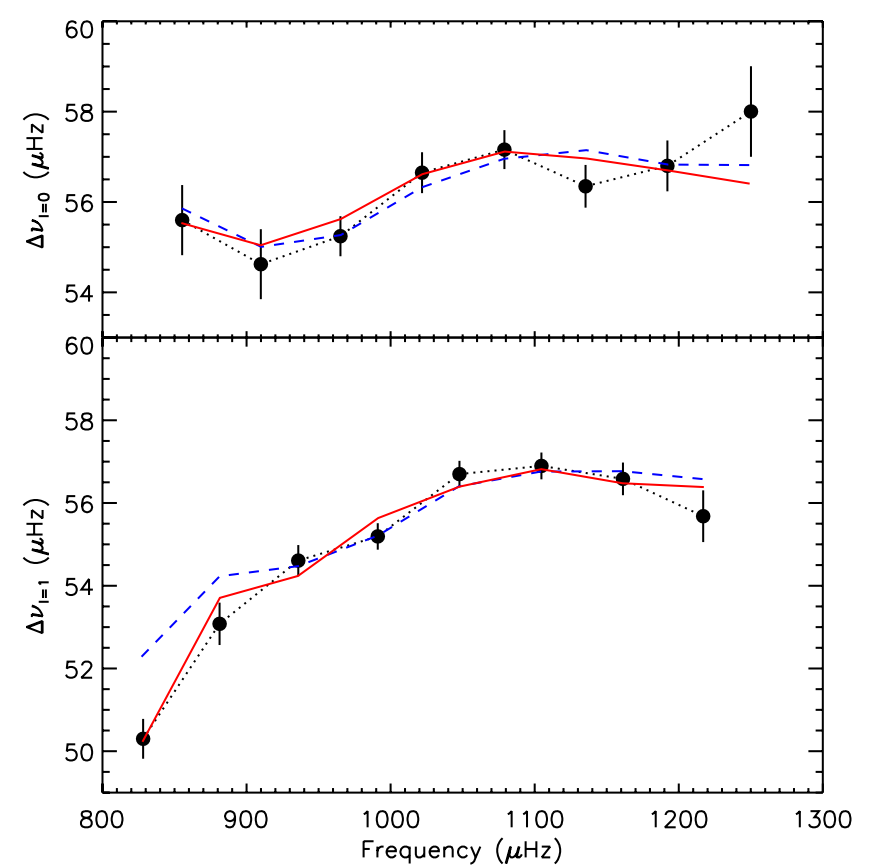

Fig. 20. Top: profiles of the $\ell=0$ large separations for the observations (black filled circles and dotted lines) and for two models that closely reproduce the oscillation due to the $\Gamma_{1}$ bump. The red solid curve corresponds to the best-fit model obtained in Sect. 5 and the blue dashed curve to a model computed with $\alpha_{\text {conv }}=0.68, Y_{0}=0.24, \alpha_{\mathrm{ov}}=0.10$, and $[Z / X]=0.14$ dex, using the GN93 mixture. Bottom: same as the top panel for the $\ell=1$ large separations.

we obtained in Sect. $2.1 .2\left(\tau_{\text {glitch }}=0.76 \pm 0.04\right)$. Figure $20 \mathrm{a}$ shows the comparison between the $\ell=0$ large separation profile of our best-fit model and that of the observations (red curve). We can see that the agreement is good, except at high frequency where the period of the oscillation appears to be shorter in the observations than in the models. However, the observations of $p$ modes over a wider range of radial orders would be required to determine whether or not this decrease in the oscillation period is significant.

Even among the models that satisfactorily fit both the position of the star in the HR diagram and the oscillation of the mode frequencies produced by the HIZ, some models yield large values of the $\chi^{2}$ function. For instance, the model represented in blue in Fig. 20 (computed with $\alpha_{\text {conv }}=0.68, Y_{0}=0.24, \alpha_{\text {ov }}=$ $0.10,[Z / X]=0.14 \mathrm{dex}$, and the mixture of GN93) reproduces the $\ell=0$ large separations quite well but the $\ell=1$ large separation profile shows some striking differences from the observations at low frequency, inducing a large value of the $\chi^{2}$ function $\left(\chi_{\text {red }}^{2}=7.44\right)$. This shows that the $\ell=1$ ridge for this model is less distorted than it is in the observations. On the basis of the conclusions drawn in Sect. 3, this means that the coupling between the p-mode cavity and the g-mode cavity is too weak.

The intensity of the $\ell=1$ ridge distortion. Since the intensity of the $\ell=1$ ridge distortion is a major contribution to the $\chi^{2}$ function (for instance, for the model plotted in blue in Fig. 20, the three lowest frequency $\ell=1$ modes account for $80 \%$ of the $\chi^{2}$ value), we tried to evaluate its dependence on the free parameters of our fit, in order to understand the results we obtained in Sect. 5. For this, we first needed to quantify the intensity of the ridge distortion caused by the avoided crossing in our models. We chose to use the frequency difference $\delta$ of the two modes that most closely surround the avoided crossing. To illustrate why these modes provide a good indicator of the ridge distortion, their frequencies were circled in Fig. 7 for both of the studied cases (weaker coupling and stronger coupling). A low value of $\delta$ indicates a weak coupling between the two cavities and a high value of $\delta$, a stronger one. For HD 49385, we have

$\delta=v_{1,12}-v_{1,11}$.

We insist that the value of $\delta$, and more generally the curvature of the $\ell=1$ ridge, can be used to estimate the strength of the coupling between the cavities only if the frequency of the avoided crossing in the models matches that of the observations (i.e. $v_{1,11}=v_{\pi_{1}}$ for HD 49385). This stresses the great interest in gaining access to the frequency of the mode that behaves mainly as a $\mathrm{g}$ mode in the avoided crossing ( $v_{1,11}$ in our case). It is also one of the reasons why we focused on finding all possible models satisfying this condition in Sect. 4.

We started from the best-fit model obtained without overshooting in Sect. 5 and varied the stellar parameters one by one. The results are shown in Fig. 21. Two striking remarks can be made about the obtained plots.

First, we observe that the intensity of the $\ell=1$ ridge distortion can account for most of the results of our fit. The quantity $\delta$ decreases when the mixing length parameter increases, as can be seen in Fig. 21a. Only small values of $\alpha_{\text {conv }}$ enable us to reproduce the observed value of $\delta\left(\delta_{\mathrm{obs}}=29.31 \pm 0.33 \mu \mathrm{Hz}\right.$, based on D10). This agrees with our fit being more consistent with a shorter mixing length. Similarly, $\delta$ decreases with increasing metallicity. We thus understand why the lower abundances of AGS05 (and more marginally the lowest metallicity with the abundances of GN93) provide a closer fit to the observations. The ridge distortion appears to be relatively independent of $Y_{0}$, and we found in Sect. 5 that $Y_{0}$ can hardly be constrained for HD 49385. Finally, the function $\delta$ depends in a more complex way on the amount of overshooting. Two families of $\alpha_{\mathrm{ov}}$ values satisfactorily reproduce $\delta_{\text {obs }}$ : very small amounts of overshooting $\left(\alpha_{\mathrm{ov}}<0.05\right)$ and moderate amounts of overshooting $\left(\alpha_{\text {ov }} \sim 0.2\right)$. This corresponds exactly to what we obtained in Sect. 5. All this establishes that the curvature of the $\ell=1$ ridge caused by the avoided crossing plays a crucial role in constraining the structure of the interior of HD 49385.

The second remark that can be made is that the curves of $\delta\left(\alpha_{\text {conv }}\right), \delta(Z / X)$, and $\delta\left(\alpha_{\text {ov }}\right)$ in Fig. 21 show a remarkable anticorrelation with the curves of $\tilde{M}\left(\alpha_{\text {conv }}\right), \tilde{M}(Z / X)$, and $\tilde{M}\left(\alpha_{\text {ov }}\right)$ shown in Figs. 13 and 16. This is most striking in the case of the variations in $\delta$ and $\tilde{M}$ with the amount of overshooting. This clearly suggests that the coupling between the cavities is inversely proportional to the stellar mass. This would explain why the mass of HD 49385 is so tightly constrained in this study. The next section is dedicated to trying to justify this phenomenon based on the interior of the computed models.

\subsection{Why are the coupling between the cavities and the stellar mass anti-correlated?}

To understand the relation between the coupling and the stellar mass, we considered two of our models for which the $\ell=1$ ridge distortion is different: model A (computed with $\alpha_{\text {conv }}=0.48$, $Y_{0}=0.24$, and $[Z / X]=0.09$ dex using the abundances of AGS05) and model B (similar to model A but with $\alpha_{\text {conv }}=0.72$ ). Model A clearly reproduces the ridge distortion $\left(\delta_{\mathrm{A}}=29.51\right.$, 0.6- $\sigma$ agreement with the observations) in contrast to model $\mathrm{B}$ $\left(\delta_{\mathrm{B}}=27.60,5.2-\sigma\right.$ agreement $)$. In agreement with the results 

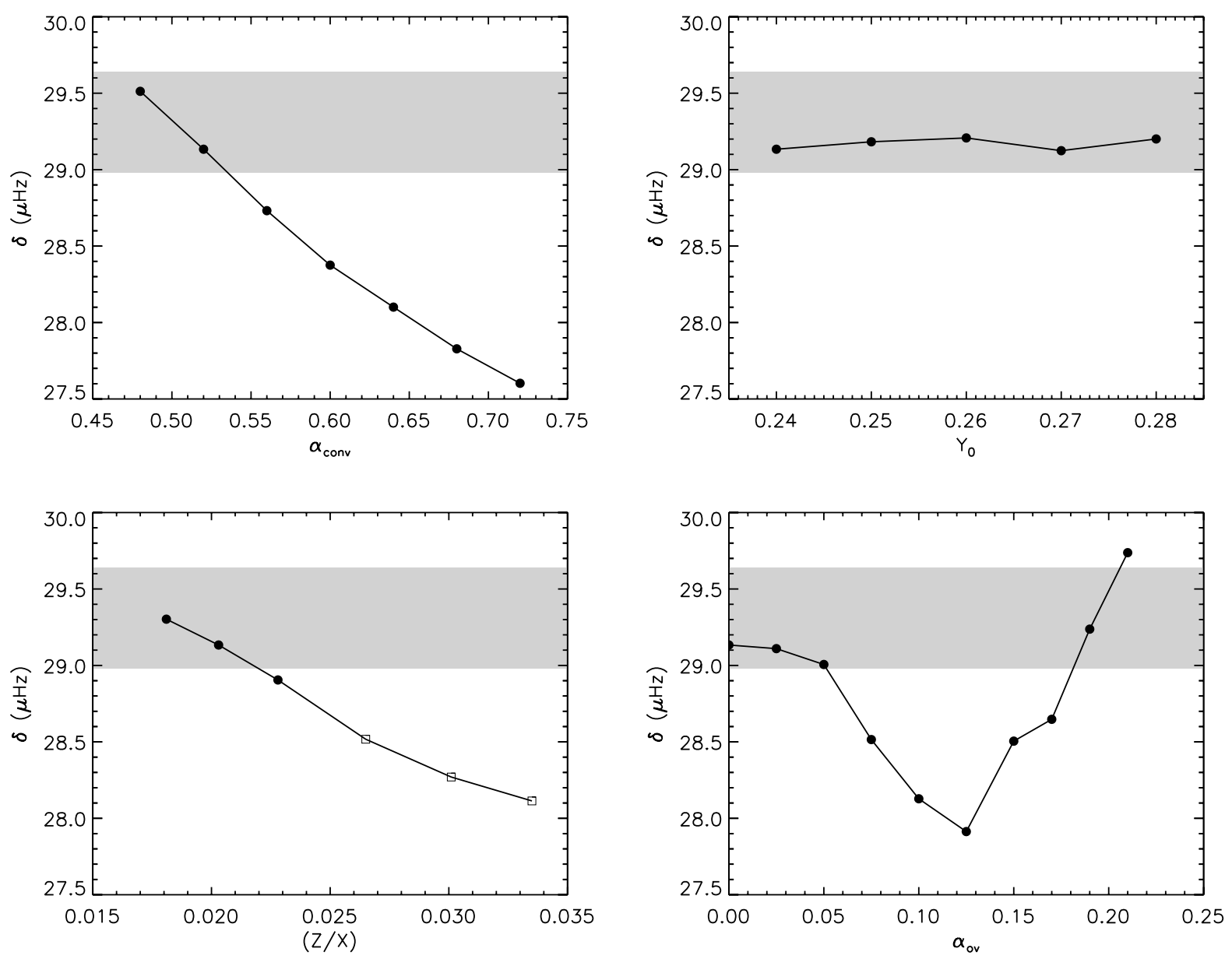

Fig. 21. Values of the quantity $\delta$ (see text) for models obtained by varying one by one the stellar parameters of the best-fit model without overshooting in Sect. 5: the mixing length parameter (top left), the initial abundance of helium (top right), the metallicity (bottom left), and the amount of overshooting (bottom right). The filled circles indicate models computed with the abundances of AGS05 and the open squares with those of GN93.

obtained in Sect. 4.5.1, model B is more massive than model A $\left(M_{\mathrm{B}}=1.37 M_{\odot}\right.$ and $\left.M_{\mathrm{A}}=1.20 M_{\odot}\right)$. We note that the results that we present in what follows remain qualitatively unchanged when considering two other models with conflicting values of $\delta$.

Figure 22a shows the propagation diagrams for models A and $\mathrm{B}$. The $\ell=1 \mathrm{Lamb}$ frequencies of the two models almost overlap in the evanescent zone, but the Brunt-Väisälä frequency of model A in this region is higher than that of model B. This confirms that the coupling between the g-mode cavity and the p-mode cavity is stronger for model A. From Eq. (13), we know that the Brunt-Väisälä frequency depends on both the temperature gradient $\nabla$ and the gradient of the mean molecular weight $\nabla \mu$. From the end of the main sequence phase to the present age, the profiles of these two quantities have been reshaped by the nuclear reactions in a shell.

Since model B is more massive than model A, the temperature in its interior is higher, which causes the nuclear reactions of the $\mathrm{CNO}$ cycle to be more efficient in the hydrogen burning shell. Figure $22 \mathrm{~b}$ shows that the peak in the nuclear reaction rate $\varepsilon_{\text {tot }}$ is indeed about twice as strong for model $\mathrm{B}$. As a result, the hydrogen content in the layer where the reactions occur is more severely depleted and the corresponding peak in the gradient of chemical composition is larger. It is therefore logical that in a small region above the hydrogen burning shell (coinciding with the evanescent zone), the gradient $\nabla \mu$ is smaller for model B (see Fig. 22c). Based on Eq. (13), this suggests that the coupling between the cavities decreases when the stellar mass increases. Similarly, since nuclear reactions are more efficient in higher-mass stars, the ratio $l / m$ (where $l(r)$ is the luminosity going through the shell of radius $r$ ) is larger for model B and therefore the temperature gradient $\nabla$ is larger in the evanescent zone. This is confirmed by Fig. 22d. This also implies that the coupling is weaker for higher-mass stars, according to Eq. (13).

\subsection{Note about the effect of microscopic diffusion}

Microscopic diffusion was not included in the grids of models presented in Sect. 5. The main reason is that the time required to compute models including diffusion is several tens times longer than when it is neglected, which would have severely limited the mesh of our grids.

However, we also computed a less dense grid of models including microscopic diffusion to determine how this process influences our results. For this, we adopted the simplified formulation of Michaud \& Proffitt (1993). Models were computed with either a small amount of overshooting $\left(\alpha_{\mathrm{ov}}=0\right)$ or a moderate one $\left(\alpha_{\mathrm{ov}}=0.2\right)$, correspondingly to the two families found in Sect. 5. We imposed an initial abundance of heavy elements such that the current surface metallicity matches the observed one. The mixing length parameter was varied from 0.48 to 0.72 (step of 0.04 ) and the initial helium abundance from 0.24 to 0.28 (step 
S. Deheuvels and E. Michel: Constraints on the structure of the core of HD 49385 via mixed modes
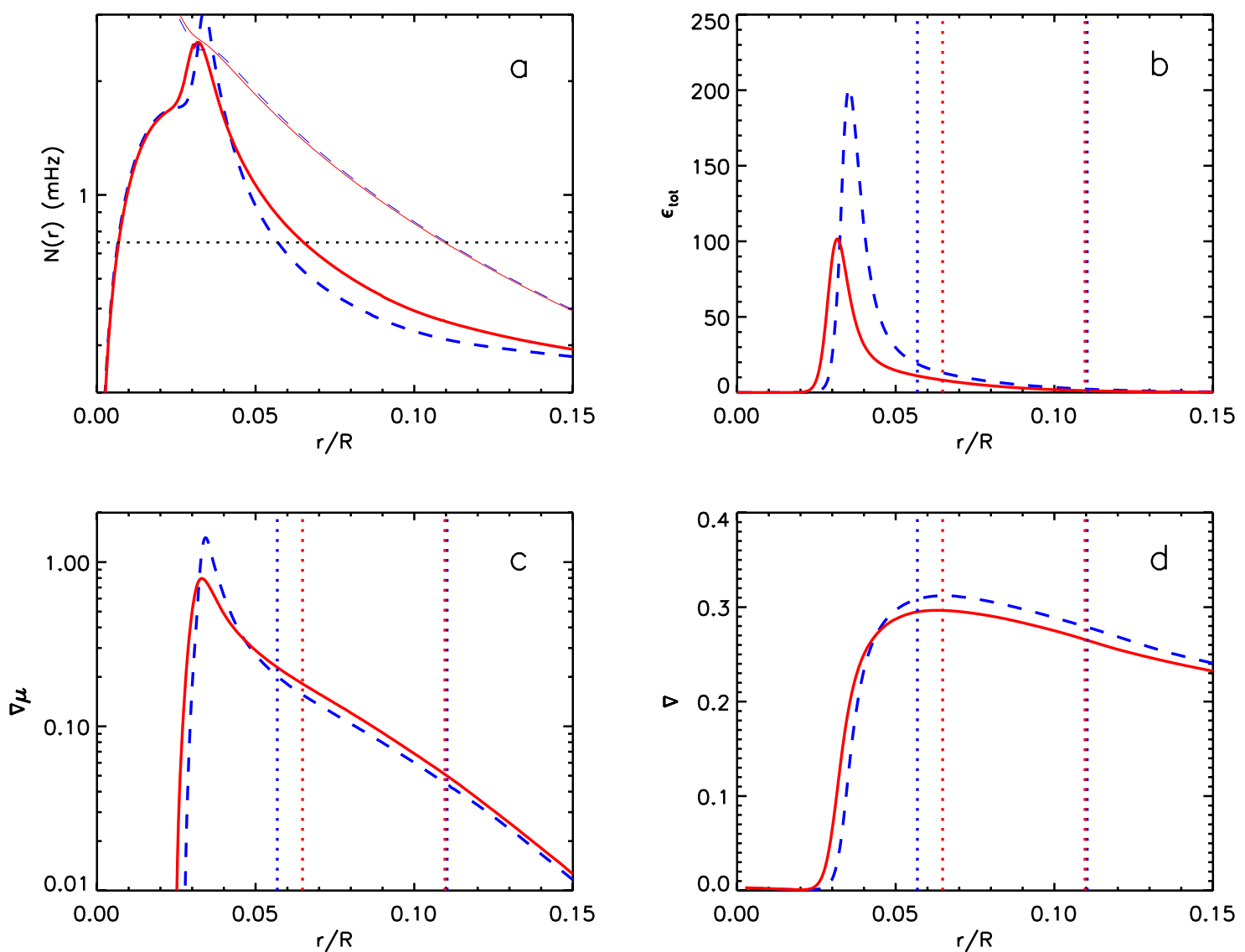

Fig. 22. Panel a) propagation diagrams of models A (red solid lines) and B (blue dashed lines). The thick lines represent the Brunt-Väisälä frequency, the thin lines the $\ell=1 \mathrm{Lamb}$ frequency. The dotted line indicates the frequency of the avoided crossing (identical for both models). We then represent the central profiles of the nuclear reaction rate $\varepsilon_{\text {tot }}(r)$ (panel b)), of the gradient of mean molecular weight $\nabla \mu$ (panel $\left.\mathbf{c}\right)$ ), and of the temperature gradient $\nabla$ (panel $\mathbf{d}$ )) for both models. The colored dotted lines indicate the boundaries of the evanescent zone for each model.

of 0.02). As before, for every set of parameters, we searched for a model satisfying condition $C$.

We again obtained a best-fit model offering a very satisfactory match to the observations $\left(\chi_{\text {red }}^{2}=2.1\right)$. All of its characteristics fall into the 1- $\sigma$ error bars obtained without diffusion, except for the age, which is smaller (4.1 Gyr for the best-fit model). The main reason why diffusion hardly changes the results is that it appears to have little influence on the coupling between the cavities (which we have shown to play a dominant role in constraining the model parameters). This can be seen in Fig. 23, which shows the propagation diagrams of two models computed with and without diffusion, both satisfying condition $C$ and sharing the same values of the free parameters. We observe that the profiles of their Brunt-Väisälä frequency almost overlap. This can be understood as follows. The largest effect of diffusion on the models is that the peak in the profile of $\nabla \mu$ caused by the withdrawal of the convective core is smoothed. This should have an impact on the coupling because $N \propto \nabla \mu$. However, this peak is rapidly reshaped by the nuclear reactions in shell. Since the center of the star is radiative, the nuclear reactions are quickly in equilibrium. The abundances of the chemical elements in the core therefore correspond to their equilibrium quantities which do not significantly vary when including diffusion. The nuclear reaction rates thus remain quite similar to the case without diffusion and so does the coupling between the cavities (see Sect. 6).

We note that interestingly, the solutions with high overshooting are found less satisfactory when including microscopic diffusion. This is probably because the models with $\alpha_{\mathrm{ov}}=0.2$ that

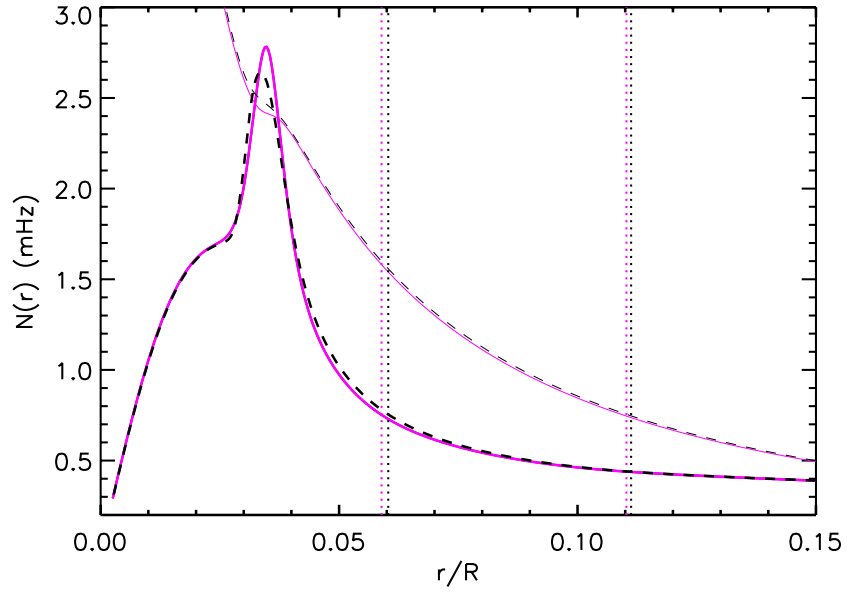

Fig. 23. Propagation diagrams of two models without microscopic diffusion (black dashed lines) and with diffusion (purple solid lines). The models both satisfy condition $C$ and share the same values of the free parameters. The symbols are the same as in Fig. 22.

satisfy condition $C$ are very close to the TAMS. Consequently, the gradient of the mean molecular weight has not yet been reshaped by the nuclear reactions in shell. A deeper analysis, which is beyond the scope of the present paper, would be required to investigate whether or not the inclusion of microscopic diffusion can rule out the solutions with a moderate amount of overshooting. 


\section{Conclusion}

We have performed a detailed seismic modeling of the solar-like pulsating subgiant HD 49385. We first investigated the possibility that the peculiarities observed in the oscillation spectrum of the star by D10 could be due to the existence of mixed modes. By extending a toy-model proposed by Christensen-Dalsgaard (2003), we have shown that $\ell=1$ avoided crossings involve more than two modes and induce a characteristic and easily recognizable distortion of the $\ell=1$ ridge in the échelle diagram. We found PoMS (post main sequence) models with an $\ell=1$ avoided crossing that can account for both the observed curvature of the ridge and the presence of one of the peaks detected outside the identified ridges. On the other hand, MS (main sequence) models fail to reproduce these features. We have thus established a firm detection of mixed modes in the spectrum of HD 49385 as well as the PoMS status of the star. We have also shown that the curvature of the $\ell=1$ ridge depends on the coupling between the p-mode and g-mode cavities and should therefore provide information about the structure of the star in the evanescent zone that separates the cavities.

We then noted that the methods usually applied to model stars needed to be adapted to the special case of stars with avoided crossings. We therefore proposed a new approach to the grid-search modeling. We first showed that, for a given physics, the combined knowledge of the frequency of an avoided crossing and of the mean large separation is enough to obtain very precise estimates of the stellar mass and age (which we have denoted $\tilde{M}$ and $\tilde{\tau}$ in this paper). We then described a method to determine $\tilde{M}$ and $\tilde{\tau}$ in a systematic way in a grid of models.

This method was applied to gain insights into how the stellar mass $\tilde{M}$ varies with the different stellar parameters and efforts were made to physically understand these variations. To model HD 49385, we used the proposed method to compute two grids of models, one assuming the heavy element abundances of GN93 (Grevesse \& Noels 1993) and the other assuming those of AGS05 (Asplund et al. 2005). In this framework, we were able to strongly constrain the mass of HD $49385(M=1.25 \pm$ $\left.0.05 M_{\odot}\right)$ and its age $(\tau=5.02 \pm 0.26 \mathrm{Gyr})$. The stellar radius was found with a precision as good as $1 \%\left(R=1.94 \pm 0.03 R_{\odot}\right)$.

We also obtained constraints on the physics of the interior of the star. Two different families of solutions were found, depending on the amount of overshooting that existed above the convective core during the main sequence phase: one with a very small amount of overshooting ( $\left.\alpha_{\text {ov }}<0.05\right)$, and the other with a moderate amount of overshooting $\left(\alpha_{\mathrm{ov}}=0.19 \pm 0.01\right)$. The models of the latter family provide the closest agreement with the observations, but they are very close to the TAMS and hence unlikely to be observed. The mixing length parameter was found to be significantly smaller than the solar one $\left(\alpha_{\text {conv }}=0.55 \pm 0.04\right.$ compared to $\alpha_{\odot}=0.64$ using the formalism of Canuto et al. 1996). Finally, we have shown that the models computed with the revised heavy-element abundances of AGS05 (Asplund et al. $2005)$ provide a closer match to the observations than the abundances of GN93 (Grevesse \& Noels 1993).

A more advanced study of the models of our grids enabled us to explain the results we obtained about the overshooting, the mixing length and the metallicity for HD 49385, in terms of stellar structure. We established that the intensity of the $\ell=$ 1 ridge distortion associated with the observed avoided crossing plays a crucial role in constraining the parameters of the models computed for HD 49385. We showed that it can account for most of the obtained results. We also found that the intensity of the coupling between the p-mode and g-mode cavities is strongly anti-correlated to the stellar mass in our models. We therefore suggested that these two quantities might be related to each other. By comparing the models of our grids, we managed to establish the link between them, mainly because the temperature and chemical composition profiles (upon which the Brunt-Väisälä frequency depends) strongly depend on the nuclear reaction rate in the hydrogen burning shell, and thus on the stellar mass. We therefore a posteriori understand why the mass is so tightly constrained in HD 49385.

The subgiant HD 49385 is the first target for which a detailed modeling was led trying to reproduce all the properties of an avoided crossing. It confirmed to a large degree that the detection of mixed modes provides unprecedented opportunities to probe the deep interior of stars. It has also given us the opportunity to show that $\ell=1$ avoided crossings have a second interest: they probe the evanescent zone by yielding an estimate of the intensity of the coupling between the p-mode and g-mode cavities. We have found that this coupling strongly depends on the stellar mass and that we can thus obtain indirect constraints on the internal structure of the star. This demonstrates that seismic observations of subgiants have the potential to contribute to ongoing debates, such as the question of the amount of core overshooting, the efficiency of convection, and the abundances of heavy elements in stars. This is all the more exciting because the space mission Kepler has claimed the detection of $\ell=1$ avoided crossings in several targets (Chaplin et al. 2010; Metcalfe et al. 2010).

Acknowledgements. This work was supported by the Centre National d'Études Spatiales (CNES).

\section{Appendix A: Location of iso- $\Delta v$ regions in the HR diagram}

We investigate the location in the HR diagram of models that have the same large separation $\Delta v$ but different masses. We consider two of these models with masses $M$ and $M^{\prime}$. Since the large separation is proportional to the square root of the mean density in the star, we have

$$
\left(\frac{\Delta v}{\Delta v^{\prime}}\right) \sim\left(\frac{M}{M^{\prime}}\right)^{1 / 2}\left(\frac{R}{R^{\prime}}\right)^{-3 / 2} \sim 1 .
$$

It has been observationally established that there exists a very tight relation between the large separation and the frequency of maximum power of the oscillations $v_{\max }$. Stello et al. (2009) found that $\Delta v \propto v_{\max }^{0.77}$. From this result, we gather that models with the same large separation also share equivalent values of $v_{\max }$. Following Brown et al. (1991), we expect $v_{\max }$ to be proportional to the acoustic cut-off frequency and thus

$$
\left(\frac{v_{\max }}{v_{\max }^{\prime}}\right) \sim\left(\frac{M}{M^{\prime}}\right)\left(\frac{R}{R^{\prime}}\right)^{-2}\left(\frac{T_{\mathrm{eff}}}{T_{\mathrm{eff}}^{\prime}}\right)^{-1 / 2} \sim 1 .
$$

By combining Eqs. (A.1) and (A.2), we obtain $R \sim T_{\mathrm{eff}}^{1 / 2}$. Since the luminosity is such that $L \sim R^{2} T_{\text {eff }}^{4}$, we conclude that the models with the same large separation satisfy

$L \propto T_{\mathrm{eff}}^{5}$

They are therefore located in the HR diagram on a line of slope equal to 5 . This property is very well-satisfied by the stellar models computed in this work. 
S. Deheuvels and E. Michel: Constraints on the structure of the core of HD 49385 via mixed modes

\section{References}

Aerts, C., Christensen-Dalsgaard, J., \& Kurtz, D. W. 2010, Asteroseismology (Berlin: Springer)

Aizenman, M., Smeyers, P., \& Weigert, A. 1977, A\&A, 58, 41

Angulo, C., Arnould, M., Rayet, M., et al. 1999, Nucl. Phys. A, 656, 3

Appourchaux, T., Gizon, L., \& Rabello-Soares, M. 1998, A\&AS, 132, 107

Asplund, M., Grevesse, N., \& Sauval, A. J. 2005, in Cosmic Abundances as

Records of Stellar Evolution and Nucleosynthesis, ed. T. G. Barnes III, \&

F. N. Bash (San Francisco: ASP), ASP Conf. Ser., 336, 25

Baglin, A., Auvergne, M., Barge, P., et al. 2006, in The CoRoT Mission, ed. M.

Fridlund, A. Baglin, J. Lochard, \& L. Conroy, Noordwijk, ESA Spec. Publ., 1306, 33

Barban, C., Michel, E., Martic, M., et al. 1999, A\&A, 350, 617

Brown, T. M., Gilliland, R. L., Noyes, R. W., \& Ramsey, L. W. 1991, ApJ, 368, 599

Canuto, V. M., Goldman, I., \& Mazzitelli, I. 1996, ApJ, 473, 550

Carrier, F., Eggenberger, P., \& Bouchy, F. 2005, A\&A, 434, 1085

Chaplin, W. J., Appourchaux, T., Elsworth, Y., et al. 2010, ApJ, 713, L169

Christensen-Dalsgaard, J. 1981, MNRAS, 194, 229

Christensen-Dalsgaard, J. 2003, Lecture Notes on Stellar Oscillations, 5th edn.

Christensen-Dalsgaard, J., \& Thompson, M. J. 1997, MNRAS, 284, 527

Deheuvels, S., \& Michel, E. 2010, Ap\&SS, 328, 259

Deheuvels, S., Bruntt, H., Michel, E., et al. 2010, A\&A, 515, A87 (D10)

Di Mauro, M. P., Christensen-Dalsgaard, J., Kjeldsen, H., Bedding, T. R., \& Paternò, L. 2003, A\&A, 404, 341

Di Mauro, M. P., Christensen-Dalsgaard, J., Paternò, L., \& D’Antona, F. 2004, Sol. Phys., 220, 185

Dziembowski, W. A., \& Pamyatnykh, A. A. 1991, A\&A, 248, L11

Gabriel, M. 1980, A\&A, 82, 8

Gai, N., Basu, S., Chaplin, W. J., \& Elsworth, Y. 2011, ApJ, 730, 63

Gelly, B., Lazrek, M., Grec, G., et al. 2002, A\&A, 394, 285
Grevesse, N., \& Noels, A. 1993, in Origin and Evolution of the Elements, ed. N. Prantzos, E. Vangioni-Flam, \& M. Casse (New York: Cambridge University Press), 15

Kippenhahn, R., \& Weigert, A. 1990, Stellar Structure and Evolution (Berlin: Springer)

Kjeldsen, H., Bedding, T. R., Viskum, M., \& Frandsen, S. 1995, AJ, 109, 1313

Kjeldsen, H., Bedding, T. R., \& Christensen-Dalsgaard, J. 2008, ApJ, 683, L175

Koch, D. G., Borucki, W. J., Basri, G., et al. 2010, ApJ, 713, L79

Lebreton, Y., Monteiro, M. J. P. F. G., Montalbán, J., et al. 2008, Ap\&SS, 316, 1

Metcalfe, T. S., Monteiro, M. J. P. F. G., Thompson, M. J., et al. 2010, ApJ, 723, 1583

Michaud, G., \& Proffitt, C. R. 1993, in Inside the Stars, ed. W. W. Weiss \& A. Baglin (San Francisco: ASP), IAU Colloq. 137, ASP Conf. Ser., 40, 246

Miglio, A., \& Montalbán, J. 2005, A\&A, 441, 615

Morel, P. 1997, A\&AS, 124, 597

Morel, P., van't Veer, C., Provost, J., et al. 1994, A\&A, 286, 91

Mosser, B., \& Appourchaux, T. 2009, A\&A, 508, 877

Osaki, J. 1975, PASJ, 27, 237

Press, W. H., Teukolsky, S. A., Vetterling, W. T., \& Flannery, B. P. 2002, Numerical recipes in $\mathrm{C}++$ : the art of scientific computing (New York: Cambridge University Press)

Provost, J., Berthomieu, G., Martić, M., \& Morel, P. 2006, A\&A, 460, 759

Roxburgh, I. W. 2009, A\&A, 506, 435

Samadi, R., Kupka, F., Goupil, M. J., Lebreton, Y., \& van't Veer-Menneret, C. 2006, A\&A, 445, 233

Scuflaire, R. 1974, A\&A, 36, 107

Scuflaire, R., Montalbán, J., Théado, S., et al. 2008, Ap\&SS, 316, 149

Stello, D., Chaplin, W. J., Basu, S., Elsworth, Y., \& Bedding, T. R. 2009, MNRAS, 400, L80

Tassoul, M. 1980, ApJS, 43, 469

von Neuman, J., \& Wigner, E. 1929, Zhurnal Phys., 30, 467 